Pacific Journal of Mathematics

MEAN VALUES OF Power SERRIS 


\title{
MEAN VALUES OF POWER SERIES
}

\author{
T. M. FLETT
}

This paper gives a unified account of a body of work on mean values of functions regular in the unit disc, relating particularly to the fractional derivatives and integrals of such functions.

Two types of fractional derivative and integral are discussed. For each of the two types of fractional derivative considered, a function analogous to the Littlewood-Paley $g$ function is defined, and the properties of these two $g$-type functions are discussed. The results obtained here include several new inequalities, and, in particular, an extension (Theorem 5) of a theorem of Hirschman for indices less than or equal to 1.

The remaining contents are as follows. In $\S 4$ the Hardy-Littlewood maximal theorem is applied to obtain an inequality for fractional derivatives. In $\$ 10$ an auxiliary theorem equivalent to one of Hardy and Littlewood is proved, and this is used to obtain a new proof of a theorem on majorants. In $\$ \$ 11-12$ new proofs of the Hardy-Littlewood theorem on fractional integrals and of some related results are given, and in $\$ 13$ a theorem of Hardy and Littlewood on the convolution series of two power series is completed and extended.

The results obtained have obvious applications in the classical theory of Fourier series, via M. Riesz's theorem on conjugate functions, but these are not stated explicitly.

2. Notation and theorems used. We assume throughout this paper that $\varphi$ is a function regular in the unit disc $\Delta=\{z \in \mathbf{C}:|z|<1\}$, and that

We write

$$
\varphi(z)=\sum_{n=0}^{\infty} c_{n} z^{n} \quad(z \in \Delta)
$$

$$
\begin{gathered}
M_{p}(\varphi ; \rho)=\left\{\frac{1}{2 \pi} \int_{-\pi}^{\pi}\left|\varphi\left(\rho e^{i \theta}\right)\right|^{p} d \theta\right\}^{1 / p} \quad(0<p<+\infty), \\
M(\varphi ; \rho)=M_{+\infty}(\varphi ; \rho)=\sup _{\theta}\left|\varphi\left(\rho e^{i \theta}\right)\right| .
\end{gathered}
$$

It is familiar that if $0<p \leqq+\infty$, then $M_{p}(\varphi ; \rho)$ increases with $\rho$, and therefore tends to a finite limit or $+\infty$ as $\rho \rightarrow 1-$. We define

$$
\mathscr{C l}_{p}(\varphi)=\lim _{\rho \rightarrow 1-} M_{p}(\varphi ; \rho) \quad(0<p \leqq+\infty),
$$

the value $+\infty$ being allowed. The class of $\varphi$ for which the limit in 
(2.1) is finite is, of course, the class $H^{p}$. It is familiar that if $\varphi \in H^{p}$, then $\varphi$ has a radial limit $\varphi\left(e^{i \theta}\right)=\lim _{\rho \rightarrow 1-} \varphi\left(\rho e^{i \theta}\right)$ for almost all $\theta$, and that

$$
\begin{gathered}
\mathscr{C}_{p}(\varphi)=\left\{\frac{1}{2 \pi} \int_{-\pi}^{\pi}\left|\varphi\left(e^{i \theta}\right)\right|^{p} d \theta\right\}^{1 / p} \quad(0<p<+\infty), \\
\mathscr{C}(\varphi)=\mathscr{C}_{+\infty}(\varphi)=\underset{\theta}{\operatorname{ess} \sup }\left|\varphi\left(e^{i \theta}\right)\right| .
\end{gathered}
$$

For any real- or complex-valued function $f$ measurable in the interval $[-\pi, \pi]$ we write

$$
\begin{gathered}
\mathscr{C}_{p}(f)=\left\{\frac{1}{2 \pi} \int_{-\pi}^{\pi}|f(\theta)|^{p} d \theta\right\}^{1 / p} \quad(0<p<+\infty), \\
\mathscr{M}(f)=\mathscr{C}_{+\infty}(f)=\text { ess sup }|f|,
\end{gathered}
$$

the value $+\infty$ being allowed. The class of $f$ for which $\mathscr{A}_{p}(f)$ is finite (where $0<p \leqq+\infty$ ) is the class $L^{p}(-\pi, \pi)$.

For any number $p$ used as an index (exponent) and such that $1<p<+\infty$, we write $p^{\prime}=p /(p-1)$, so that $p$ and $p^{\prime}$ are conjugate indices in the sense of Hölder's inequality. We extend this notation to include $p=1$ and $p=+\infty$ by interpreting $1 / 0$ as $+\infty$ and $1 /+\infty$ as 0 . All indices and other parameters are assumed to be finite except where otherwise stated.

Any inequality $L \leqq R$ quoted or proved is to be interpreted as meaning ' if $R$ is finite, then $L$ is finite, and $L \leqq R$ '.

We use $A(b, c, \cdots)$ to denote a positive constant depending only on $b, c, \cdots$, not necessarily the same on any two occurrences; $A$ by itself will denote a positive absolute constant. We also sometimes write $B$ for constants of the form $A(b, c, \cdots)$; these too are not necessarily the same on any two occurrences.

We collect together here a number of known theorems which we use in the course of our proofs.

Theorem A. Let

$$
p \geqq 1, \quad q \geqq 1, \quad \frac{1}{r}=\frac{1}{p}+\frac{1}{q}-1 \geqq 0 ，
$$

let $f, g$ be real- or complex-valued functions measurable on $[-\pi, \pi]$, and let

$$
h(\theta)=\frac{1}{2 \pi} \int_{-\pi}^{\pi} f(\theta-t) g(t) d t .
$$

Then

$$
\mathscr{C}_{r}(h) \leqq \mathscr{C l}_{p}(f) \mathscr{C l}_{q}(g) .
$$


This is a well-known inequality of W. H. Young (see, for example, [22, i. p. 37]).

THEOREM B. Let $f$ be a function measurable on the interval 10 , $+\infty\left[\right.$, let $f(x) \geqq 0$ for $x>0$, and let $F_{\delta}(x)$ be the RiemannLiouville integral of $f$ of order $\delta$ with origin 0 , i.e.

$$
F_{\delta}(x)=\frac{1}{\Gamma(\delta)} \int_{0}^{x}(x-y)^{\delta-1} f(y) d y .
$$

If $\lambda>-1$ and either

$$
k \geqq l \geqq 1, \delta>1 / l-1 / k, \text { or } k>l>1, \quad \delta=1 / l-1 / k,
$$

then

$$
\left\{\int_{0}^{+\infty} x^{-1-k \lambda-k \delta} F_{\delta}^{k}(x) d x\right\}^{1 / k} \leqq A(k, l, \delta, \lambda)\left\{\int_{0}^{+\infty} x^{-1-l \lambda} f^{l}(x) d x\right\}^{1 / l} .
$$

For $\delta>1 / l-1 / k$ this is essentially an elementary application of Hölder's inequality; for $\delta=1 / l-1 / k$, the result lies deeper, the case $\lambda=-1 / l$ being the Hardy-Littlewood theorem on fractional integrals of real functions (see [5, Th. 2]).

THeorem C. If $\varphi \in H^{p}$, where $0<p \leqq+\infty$, then $\varphi$ can be expressed in the form $\varphi=\varphi_{1}+\varphi_{2}$, where $\varphi_{1}$ and $\varphi_{2}$ are regular and have no zeros in 4 , and

$$
\mathscr{M}_{p}\left(\varphi_{i}\right) \leqq 2 \mathscr{C l}_{p}(\varphi) \quad(i=1,2) .
$$

This is a familiar theorem of Hardy and Littlewood ([8, p. 207]).

Theorem D. If $0<p \leqq+\infty$ and $\mu=\max \{0,1 / p-1\}$, then

$$
n^{-\mu}\left|c_{n}\right| \leqq A(p) \mathscr{L l}_{p}(\varphi) \text {. }
$$

This also is due to Hardy and Littlewood ([12, Theorem 28]).

Theorem E. Let $0<\eta<1$, and let $S(\theta)=S_{\eta}(\theta)$ be the open subset of $\Delta$ bounded by the two tangents from the point $e^{i \theta}$ to the circle with centre 0 and radius $\eta$, together with the longer arc of this circle between the points of contact. Let also $\varphi$ be regular in 4 , and let

$$
\Phi(\theta)=\sup _{z \in S(\theta)}|\varphi(z)| .
$$

Then for $0<p \leqq+\infty$

$$
\mathscr{C}_{p}(\Phi) \leqq A(p, \eta) \mathscr{C}_{p}(\varphi) .
$$


This is the Hardy-Littlewood 'Complex Max' theorem (see, for example, [22, i, p. 278]).

THEOREM F. Let $\varphi$ be regular in $\Delta$, and let

$$
T_{k, \sigma}(\theta)=\left\{\int_{0}^{1}(1-\rho)^{\sigma+k-2} d \rho \int_{-\pi}^{\pi} \frac{\left|\varphi^{\prime}\left(\rho e^{i \theta-i t}\right)\right|^{k}}{\left|1-\rho e^{i t}\right|^{\sigma}} d t\right\}^{1 / k} .
$$

If $p>0, k \geqq 2, \sigma>\max \{1, k / p\}$, then

$$
\mathscr{C l}_{p}\left(T_{k, \sigma}\right) \leqq A(k, p, \sigma) \mathscr{C l}_{p}(\varphi) .
$$

This is one of the consequences of the Littlewood-Paley $g$-theorem (see [3, Th. 15]).

THEOREM G. Let $f \in L^{p}(-\pi, \pi)$, where $p>1$, let the complex Fourier series of $f$ be $\sum_{-\infty}^{\infty} \gamma_{n} e^{n i \theta}$, and let $\psi(z)=\sum_{n=1}^{\infty} \gamma_{n} z^{n}(z \in \Delta)$. Then

$$
\mathscr{C l}_{p}(\psi) \leqq A(p) \mathscr{C l}_{p}(f) \text {. }
$$

This is equivalent to M. Riesz's theorem on conjugate functions (see Hardy and Littlewood [9] for further explanations).

In addition to these theorems we also make extensive use of Hölder's inequality, and of Minkowski's inequality in the form

$$
\left\{\int g(x) d x\left\{\int f(x, y) h(y) d y\right\}^{k}\right\}^{1 / k} \leqq \int h(y) d y\left\{\int f^{k}(x, y) g(x) d x\right\}^{1 / k},
$$

where $k \geqq 1$, and $f, g, h$ are nonnegative. We use also the analogous result for $k=+\infty$, namely

$$
\sup _{x}\left\{\int f(x, y) h(y) d y\right\} \leqq \int\left\{\sup _{x} f(x, y)\right\} h(y) d y \text {. }
$$

3. Fractional derivatives and integrals, first type. The definition of fractional derivative and integral which is used in $\S \S 3-13$ is as follows. Let $\varphi$ be defined as in $\S 2$, i.e. $\varphi$ is regular in $\Delta$, and

$$
\varphi(z)=\sum_{n=0}^{\infty} c_{n} z^{n} \quad(z \in \Delta) .
$$

Then for any $\beta \geqq 0$ the fractional derivative $\vartheta^{\beta} \varphi$ of $\varphi$ of order $\beta$ is given by

$$
\vartheta^{\beta} \varphi(z)=\sum_{n=0}^{\infty} n^{\beta} c_{n} z^{n} \quad(z \in \Delta) .
$$

Clearly $\vartheta^{\beta} \varphi$ is regular in $\Delta$, and

$$
\vartheta^{\beta}\left(\vartheta^{r} \varphi\right)=\vartheta^{\beta+r} \varphi
$$


for all nonnegative $\beta, \gamma$.

The corresponding definition of the fractional integral applies only to functions vanishing at the origin. Thus if $\phi(0)=c_{0}=0$, then for any $\alpha \geqq 0$ the fractional integral $\vartheta_{\alpha} \varphi$ of $\phi$ of order $\alpha$ is given by

$$
\vartheta_{\alpha} \varphi(z)=\sum_{n=1}^{\infty} n^{-\alpha} c_{n} z^{n} \quad(z \in \Delta) .
$$

As for the fractional derivative, the fractional integral $\vartheta_{\alpha} \varphi$ is regular in $A$, and

$$
\vartheta_{\alpha}\left(\vartheta_{\gamma} \varphi\right)=\vartheta_{\alpha+\gamma} \varphi
$$

for all nonnegative $\alpha, \gamma$.

When $\varphi(0)=0,(3.1)$ and (3.3) can be used to define $\vartheta_{\alpha} \varphi$ and $\vartheta^{\beta} \varphi$ for all real $\alpha, \beta$ (so that $\vartheta_{\alpha} \varphi=\vartheta^{-\alpha} \varphi$ for all real $\alpha$ ), and then (3.2) and (3.4) hold for all real $\alpha, \beta, \gamma$.

The functions $\vartheta_{\alpha} \varphi$ and $\vartheta^{\beta} \varphi$ defined above seem to have been first studied by Hadamard [7] $]^{1}$. For $\alpha>0, i^{-\alpha} \vartheta_{\alpha} \varphi\left(\rho e^{i \theta}\right)$ is the Weyl fractional integral of order $\alpha$ of the function $\theta m \varphi\left(\rho e^{i \theta}\right)$, and for any positive integer $m$

$$
i^{m} \vartheta^{m} \varphi\left(\rho e^{i \theta}\right)=\frac{\partial^{m}}{\partial \theta^{m}} \varphi\left(\rho e^{i \theta}\right) .
$$

Thus the definitions (3.1) and (3.3) correspond roughly to differentiation and integration with respect to $\theta$. We note also that if $m$ is a positive integer, then

$$
\vartheta^{m} \varphi(z)=\left(z \frac{d}{d z}\right)^{m} \varphi(z)
$$

so that $\vartheta^{1}$ has its traditional meaning of $z \frac{d}{d z}$.

For $\alpha>0$ the fractional integral $\vartheta_{a} \varphi$ is connected with $\varphi$ by the relation

$$
\vartheta_{\alpha} \varphi\left(\rho e^{i \theta}\right)=\frac{1}{\Gamma(\alpha)} \int_{0}^{\rho}\left(\log \frac{\rho}{\sigma}\right)^{\alpha-1} \varphi\left(\sigma e^{i \theta}\right) \frac{d \sigma}{\sigma},
$$

where $0<\rho<1$; this relation is easily obtained by term-by-term integration, using the formulae

$$
\rho^{-n} \int_{0}^{\rho}(\log \rho / \sigma)^{\alpha-1} \sigma^{n-1} d \sigma=\int_{0}^{1}(\log 1 / s)^{\alpha-1} s^{n-1} d s=\int_{0}^{+\infty} t^{\alpha-1} e^{-n t} d t=n^{-\alpha} \Gamma(\alpha),
$$

1 Hadamard writes $\mathscr{D}_{z}^{\beta}$ in place of our $\vartheta^{\beta}$ (for all real $\beta$ ). We have followed Hardy and Littlewood in using inferior letters for integrals and superior letters for derivatives. 
where $\alpha>0, n>0, \rho>0$.

The formula (3.6) was obtained by Hadamard [7, p. 157], but does not seem to have been used by subsequent writers on fractional derivatives and integrals. In $\$ \S 4-12$ we develop the theory of the functions $\vartheta^{\beta} \varphi$ and $\vartheta_{\alpha} \varphi$, making systematic use of the formula (3.6).

4. As our first application of Hadamard's formula (3.6), we prove:

THEOREM 1. Let $S_{\eta}(\theta)$ be the kite-shaped region defined in Theorem $E$, where $0<\eta<1$, let

$$
\Phi(\theta)=\sup _{z \in S_{\eta}(\theta)}|\varphi(z)|,
$$

and let $\beta>0$. Then for $0 \leqq \rho<1$

$$
\left|\vartheta^{\beta} \varphi\left(\rho e^{i \theta}\right)\right| \leqq A(\beta, \eta) \rho(1-\rho)^{-\beta} \Phi(\theta) \text {. }
$$

A similar result for a different type of fractional derivative is proved by Hardy and Littlewood [17, Th. 5] (see also Hirschman [18, Lemma 4.1], and Flett [6, Th. 8]).

Suppose first that $\beta$ is a positive integer, $m$ say, and let $C$ be the circle with centre $z=\rho e^{i \theta}$ and radius $\frac{1}{2} \eta(1-\rho)$. By (3.5), for $z \neq 0$ we have

$$
z^{-1} \vartheta^{m} \varphi(z)=z^{-1}\left(z \frac{d}{d z}\right)^{m} \varphi(z)=\frac{1}{2 \pi i} \int_{C} \frac{P(\zeta, z) \varphi(\zeta)}{(\zeta-z)^{m+1}} d \zeta,
$$

where $P$ is a polynomial of degree $m-1$ in $\zeta, z$ depending only on $m$. Since $C \subset S_{\eta}(\theta)$, it follows that

$$
\rho^{-1}\left|\vartheta^{m} \varphi\left(\rho e^{i \theta}\right)\right| \leqq A(\beta, \eta)(1-\rho)^{-m} \Phi(\theta),
$$

and this implies (4.1), since $\vartheta^{m} \varphi(0)=0$.

Next, let $\beta$ be nonintegral, and let $m=[\beta]+1$ (where $[\beta]$ denotes, as usual, the integral part of $\beta)$. Since $\vartheta^{\beta} \varphi=\vartheta_{m-\beta}\left(\vartheta^{m} \varphi\right)$, (3.6) gives

$$
\vartheta^{\beta} \varphi\left(\rho e^{i \theta}\right)=\frac{1}{\Gamma(m-\beta)} \int_{0}^{\rho}\left(\log \frac{\rho}{\sigma}\right)^{m-\beta-1} \vartheta^{m} \varphi\left(\sigma e^{i \theta}\right) \frac{d \sigma}{\sigma},
$$

and since $\log 1 / x \geqq 1-x$ for $x>0$, and $m-\beta \leqq 1$, we obtain from (4.3) and (4.2) that

$$
\left|\vartheta^{\beta} \varphi\left(\rho e^{i \theta}\right)\right| \leqq A(\beta, \eta) \Phi(\theta) \rho^{-m+\beta+1} \int_{0}^{\rho}(\rho-\sigma)^{m-\beta-1}(1-\sigma)^{-m} d \sigma .
$$

On substituting $\sigma=1-(1-\rho) x$, we see that the integral on the right is equal to 


$$
(1-\rho)^{-\beta} \int_{1}^{1 /(1-\rho)}(x-1)^{m-\beta-1} x^{-m} d x \leqq(1-\rho)^{-\beta} \int_{1}^{+\infty}=A(\beta)(1-\rho)^{-\beta},
$$

and (4.4) and (4.5) together imply (4.1) for $1 / 2 \leqq \rho<1$. On the other hand, if $0<\rho<1 / 2$, then the integral on the right of (4.4) does not exceed

$$
2^{m} \int_{0}^{\rho}(\rho-\sigma)^{m-\beta-1} d \sigma=2^{m} \rho^{m-\beta} /(m-\beta) \leqq A(\beta) \rho^{m-\beta}(1-\rho)^{-\beta},
$$

and again the inequality (4.1) follows.

Theorem 1. Corollary 1. If $0<p \leqq+\infty, \beta>0$, then for $0 \leqq \rho<1$

$$
M_{p}\left(\vartheta^{\beta} \varphi ; \rho\right) \leqq A(p, \beta) \rho(1-\rho)^{-\beta} \mathscr{C}_{p}(\varphi) .
$$

This follows from the main theorem and Theorem $\mathrm{E}$, with $\eta=1 / 2$ (say). Applying this corollary to the function $z \sim \varphi\left(\rho^{1 / 2} z\right)$, we deduce also

Theorem 1. Corollary 2. Let $0<p \leqq+\infty$, and let

$$
M_{p}(\varphi ; \rho) \leqq c(\rho) \quad(0 \leqq \rho<1) .
$$

Then for $\beta>0$

$$
M_{p}\left(\vartheta^{\beta} \varphi ; \rho\right) \leqq A(p, \beta) \rho^{1 / 2}(1-\rho)^{-\beta} c\left(\rho^{1 / 2}\right) \quad(0 \leqq \rho<1) .
$$

5. Theorems of Littlewood-Paley type. We consider next a group of three theorems closely related to results of Littlewood and Paley, Hirschman, and the author.

For any $\varphi$ regular in the unit disc $\Delta$, and for any positive $k$ and $\beta$, let

$$
\mathscr{G}_{k, \beta}(\theta)=\left\{\int_{0}^{1}\left(\log \frac{1}{\rho}\right)^{k \beta-1}\left|\vartheta^{\beta} \varphi\left(\rho e^{i \theta}\right)\right|^{k} \frac{d \rho}{\rho}\right\}^{1 / k}
$$

THeOREM 2. If $\beta>0$, and either $k \geqq l \geqq 1, \delta>1 / l-1 / k$, or $k>l>1, \delta=1 / l-1 / k$, then for each $\theta$

$$
\mathscr{G}_{k, \beta}(\theta) \leqq A(k, l, \beta, \delta) \mathscr{G} l, \beta+\delta(\theta) .
$$

In particular, if $k \geqq 1$ and $\gamma>\beta>0$, then for each $\theta$

$$
\mathscr{C}_{k, \beta}(\theta) \leqq A(k, \beta, \gamma) \mathscr{C}_{k, \gamma}(\theta) \text {. }
$$

THEOREM 3. If $p>0, k \geqq 2, \beta>0$, then 


$$
\mathscr{C}_{p}\left(\mathscr{G}_{k, \beta}\right) \leqq A(k, p, \beta) \mathscr{C}_{p}(\varphi) .
$$

THEOREM 4. If $p>1,1<k \leqq 2, \beta>0$, and $\varphi(0)=0$, then

$$
\mathscr{C l}_{p}(\varphi) \leqq A(k, p, \beta) \mathscr{C l}_{p}\left(\mathscr{G}_{k, \beta}\right) \text {. }
$$

The results of Theorems 3 and 4 with $\mathscr{G}_{k, \beta}$ replaceb by the function $g_{k, \beta}$ given by

$$
g_{h, \beta}(\theta)=\left\{\int_{0}^{1}(1-\rho)^{k, \beta-1} \rho^{-k}\left|\vartheta^{\beta} \varphi\left(\rho e^{i \theta}\right)\right|^{k} d \rho\right\}^{1 / k}
$$

are already known. The cases $k=2, \beta=1$ and $k=p, \beta=1$ of these results for $g_{k, \beta}$ were proved by Littlewood and Paley [19], the function $g_{2,1}$ being the well-known Littlewood-Paley $g$-function The remaining cases where $\beta=1$ are due to Marcinkiewicz and Zygmund [20], and the cases where $\beta \neq 1$ are due to Hirschman [18] and the author $[4,6]$. The crucial result for these theorems for $g_{k, \beta}$ is that for $g_{2,1}$ corresponding to Theorem 3 (i.e. the Littlewood-Paley $g$-theorem), all the other results being obtainable from this.

It is easy to pass from (5.3) to the corresponding inequality for $g_{k, \beta}$ and vice-versa, for it is obvious that if $k>1, \beta>0$, then

$$
\mathscr{G}_{k, \beta}(\theta) \leqq A(k, \beta) g_{k, \beta}(\theta),
$$

and in virtue of Theorem 1, we have also

$$
g_{k, \beta}^{k}(\theta)=\int_{0}^{1 / 2}+\int_{1 / 2}^{1} \leqq A(k, \beta) \Phi^{k}(\theta)+A(k, \beta) \mathscr{C}_{k, \beta}^{k}(\theta)
$$

for $k>0, \beta>0$. It is also not difficult to deduce Theorem 4 from the result for $g_{k, \beta}$ corresponding to Theorem 3. However, the arguments involved in the proofs of these various results, at least for $\beta \neq 1$, apply much more naturally to $\mathscr{S}_{k, \beta}$ than to $g_{k, \beta}$, and it seems worth while to give independent proofs of Theorems 3 and 4 .

The inequality (5.1) is new. It shows in particular that the cases $k \neq 2, \beta=1$ of Theorems 3 and 4 are implied by the cases $k=2$ of these results, and thus provides a new proof of the results of Marcinkiewicz and Zygmund mentioned above. The simple special case (5.2) also enables us to reduce the proof of Theorem 3 to the case where $\beta$ is a positive integer, and this in turn simplifies one of the estimates involved.

6. We begin with the proof of Theorem 2. If $\beta>0, \delta>0$ then $\vartheta^{\beta} \varphi=\vartheta_{\hat{o}}\left(\vartheta^{\beta+\hat{\delta}} \varphi\right)$, so that, by $(3.6)$,

$$
\left|\vartheta^{\beta} \varphi\left(\rho e^{i \theta}\right)\right| \leqq \frac{1}{\Gamma(\delta)} \int_{0}^{\rho}\left(\log \frac{\rho}{\sigma}\right)^{\delta-1}\left|\vartheta^{\beta+\delta} \varphi\left(\sigma e^{i \theta}\right)\right| \frac{d \sigma}{\sigma} .
$$


The required inequality (5.1) is therefore a consequence of the following lemma.

LEMma 1. Let $h$ be a function measurable on the interval ]0,1[, let $h(\rho) \geqq 0$ for $0<\rho<1$, and let

$$
h_{\hat{o}}(\rho)=\frac{1}{\Gamma(\delta)} \int_{0}^{\rho}\left(\log \frac{\rho}{\sigma}\right)^{\delta-1} h(\sigma) \frac{d \sigma}{\sigma} .
$$

If $\beta>0$, and either $k \geqq l \geqq 1, \delta>1 / l-1 / k$, or $k>l>1, \delta=1 / l-1 / k$, then

$$
\left\{\int_{0}^{1}\left(\log \frac{1}{\rho}\right)^{k \beta-1} h_{\delta}^{k}(\rho) \frac{d \rho}{\rho}\right\}^{1 / k} \leqq A(k, l, \beta, \delta)\left\{\int_{0}^{1}\left(\log \frac{1}{\rho}\right)^{l \beta+l \delta-1} h^{l}(\rho) \frac{d \rho}{\rho}\right\}^{1 / l} .
$$

This follows easily from Theorem B by the transformation

$$
1 / x=\log 1 / \rho, \quad 1 / y=\log 1 / \sigma, \quad f(x)=x^{-\delta-1} h\left(e^{-1 / x}\right), \quad \lambda=\beta-1 .
$$

The lemma may also be proved independently of Theorem B. In our arguments we make essential use only of the case $k=l$ (this gives the inequality (5.2)), and since the direct proof of this case of the lemma is particularly simple, we give it here for the sake of completeness.

Let $k \geqq 1, \beta>0, \delta>0$, and choose $\mu$, depending on $k, \beta, \delta$, such that $\delta / k^{\prime}<\mu<\beta+\delta / k^{\prime}$. For $k>1$ we have, by Hölder's inequality,

$$
\begin{aligned}
& \left\{\Gamma(\delta) h_{\delta}(\rho)\right\}^{k} \\
& \leqq\left\{\int_{0}^{\rho}\left(\log \frac{1}{\sigma}\right)^{k \mu}\left(\log \frac{\rho}{\sigma}\right)^{\delta-1} h^{k}(\sigma) \frac{d \sigma}{\sigma}\right\}\left\{\int_{0}^{\rho}\left(\log \frac{1}{\sigma}\right)^{-k^{\prime} \mu}\left(\log \frac{\rho}{\sigma}\right)^{\delta-1} \frac{d \sigma}{\sigma}\right\}^{k / k^{\prime}} \\
& =A(k, \beta, \delta)\left(\log \frac{1}{\rho}\right)^{k \delta / k^{\prime}-k \mu} \int_{0}^{\rho}\left(\log \frac{1}{\sigma}\right)^{k \mu}\left(\log \frac{\rho}{\sigma}\right)^{\delta-1} h^{k}(\sigma) \frac{d \sigma}{\sigma}
\end{aligned}
$$

the second factor on the right of the first line of (6.1) being easily evaluated by means of the substitution $1 / x=\log 1 / \rho, 1 / y=\log 1 / \sigma$. If $k=1$, the final inequality in $(6.1)$ holds trivially (where $1 / k^{\prime}$ is interpreted as 0 ). Writing $c=k \beta+k \delta / k^{\prime}-k \mu$, we therefore have for $k \geqq 1$

$$
\begin{aligned}
& \int_{0}^{1}\left(\log \frac{1}{\rho}\right)^{k \beta-1} h_{\delta}^{k}(\rho) \frac{d \rho}{\rho} \\
& \leqq A(k, \beta, \delta) \int_{0}^{1}\left(\log \frac{1}{\rho}\right)^{c-1} \frac{d \rho}{\rho} \int_{0}^{\rho}\left(\log \frac{1}{\sigma}\right)^{k \mu}\left(\log \frac{\rho}{\sigma}\right)^{\delta-1} h^{k}(\sigma) \frac{d \sigma}{\sigma} \\
& \quad=A(k, \beta, \delta) \int_{0}^{1}\left(\log \frac{1}{\sigma}\right)^{k \mu} h^{k}(\sigma) \frac{d \sigma}{\sigma} \int_{\sigma}^{1}\left(\log \frac{1}{\rho}\right)^{c-1}\left(\log \frac{\rho}{\sigma}\right)^{\delta-1} \frac{d \rho}{\rho} .
\end{aligned}
$$


On substituting $s=\log 1 / \rho, t=\log 1 / \sigma$ we see that the inner integral on the right of (6.2) is equal to $A(k, \beta, \delta)(\log 1 / \sigma)^{c+\delta-1}$, and the result now follows.

7. We take next the proof of Theorem 3 , and here we use Theorem $\mathrm{F}$ (so that the proof, like that for $g_{k, \beta}$, depends ultimately on the Littlewood-Paley $g$-theorem).

As remarked above, it is enough to prove (5.3) when $\beta$ is a large integer. We note now that if $E(z)=1 /(1-z)$ then

$$
\vartheta^{\beta} \varphi\left(\rho^{2} e^{i \theta}\right)=\frac{1}{2 \pi} \int_{-\pi}^{\pi} \rho e^{i \theta-i t} \varphi^{\prime}\left(\rho e^{i \theta-i t}\right) \vartheta^{\beta-1} E\left(\rho e^{i t}\right) d t .
$$

It is immediate from (3.5) that for positive integral $\beta .^{2}$

$$
\left|\vartheta^{\beta-1} E\left(\rho e^{i t}\right)\right| \leqq A(\beta) \rho\left|1-\rho e^{i t}\right|^{-\beta},
$$

and therefore also

$$
\begin{aligned}
\rho^{-2 k}\left|\vartheta^{\beta} \varphi\left(\rho^{2} e^{i \theta}\right)\right|^{k} & \leqq A(\beta)\left\{\int_{-\pi}^{\pi} \frac{\left|\varphi^{\prime}\left(\rho e^{i \theta-i t}\right)\right|}{\left|1-\rho e^{i t}\right|^{\beta}} d t\right\}^{k} \\
& \leqq A(\beta)\left\{\int_{-\pi}^{\pi} \frac{\left|\varphi^{\prime}\left(\rho e^{i \theta-i t}\right)\right|^{k}}{\left|1-\rho e^{i t}\right|^{k \beta-2 k+2}} d t\right\}\left\{\int_{-\pi}^{\pi} \frac{d t}{\left|1-\rho e^{i t}\right|^{2}}\right\}^{k-1} \\
& =A(k, \beta)\left(1-\rho^{2}\right)^{1-k} \int_{-\pi}^{\pi} \frac{\left|\varphi^{\prime}\left(\rho e^{i \theta-i t}\right)\right|^{k}}{\left|1-\rho e^{i t}\right|^{k \beta-2 k+2}} d t .
\end{aligned}
$$

Replacing $\rho$ by $\rho^{2}$ in the integral for $\mathscr{G}_{k, \beta}^{k}$, and noting that

$$
(\log 1 / \rho)^{k \beta-1} \rho^{2 k-1} \leqq A(k, \beta)(1-\rho)^{k \beta-1},
$$

we thus obtain (again for positive integral $\beta$ )

$$
\mathscr{G}_{k, \beta}(\theta) \leqq A(k, \beta) T_{k, k \beta-2 k+2}(\theta),
$$

where $T$ is defined as in Theorem F. Since we may assume that $k \beta-2 k+2>\max \{1, k / p\}$, the required inequality follows from Theorem $\mathrm{F}$.

When $p=+\infty$, the inequality (5.3) is known to be false for $\beta=1$ (take $\left.\varphi(z)=(1-z)^{i}\right)$, and is almost certainly false for all $\beta>0$.

8. For the proof of Theorem 4 we use an argument of a type first employed by Littlewood and Paley for the case $\beta=1$ of the $g_{k, \beta}$-theorems, and subsequently extended by Hirschman [18] and the author [6] to the case $\beta \neq 1$. For $\mathscr{G}_{k, \beta}$, the argument takes a very symmetrical form.

2 The inequality (7.1) continues to hold for nonintegral $\beta>0$, but its proof for such $\beta$ is less trivial (see [4, p. 378]). 
To prove Theorem 4, it is enough to show that if $p>1,1<k \leqq 2$, $\beta>0$, then

$$
M_{p}(\varphi ; R) \leqq A(k, p, \beta) \mathscr{C}_{p}\left(\mathscr{G}_{k, \beta}\right)
$$

for $0<R<1$. Since the expression on the left of (8.1) is equal to

$$
\sup \left|\frac{1}{2 \pi} \int_{-\pi}^{\pi} \varphi\left(R e^{i \theta}\right) V(\theta) d \theta\right|,
$$

where the supremum is taken over all complex-valued trigonometric polynomials $V$ satisfying $\mathscr{C}_{p^{\prime}}(V)=1$, it is therefore enough to prove that for any such $V$

$$
\left|\frac{1}{2 \pi} \int_{-\pi}^{\pi} \varphi\left(R e^{i \theta}\right) V(\theta) d \theta\right| \leqq A(k, p, \beta) \mathscr{C}_{p}\left(\mathscr{G}_{k, \beta}\right)
$$

when $0<R<1$.

Let $V(\theta)=\sum_{n=-N}^{N} \kappa_{n} e^{n i \theta}$, let $\xi(z)=\sum_{n=1}^{N} \kappa_{-n} z^{n}$, and for any $\gamma>0$ and $0<R<1$ let

$$
\mathscr{C}_{k^{\prime}, \gamma}(\theta)=\left\{\int_{0}^{1}\left(\log \frac{1}{\rho}\right)^{k^{\prime} \gamma-1}\left|\vartheta^{r} \xi\left(R \rho e^{i \theta}\right)\right|^{k^{\prime}} \frac{d \rho}{\rho}\right\}^{1 / k^{\prime}} .
$$

By Theorem 3 and Theorem G,

$$
\begin{aligned}
\mathscr{C}_{p^{\prime}}\left(\mathscr{C}_{k^{\prime}, \gamma}\right) & \leqq A(k, p, \gamma) M_{p^{\prime}}(\xi ; R) \leqq A(k, p, \gamma) \mathscr{C}_{p^{\prime}}(V) \\
& =A(k, p, \gamma) .
\end{aligned}
$$

We note now that

$$
\int_{-\pi}^{\pi} \varphi\left(R e^{i \theta}\right) V(\theta) d \theta=2 \pi \sum_{n=1}^{N} c_{n} \kappa_{-n} R^{n},
$$

and hence, by the formulae (3.7), for any positive $\beta, \gamma$,

$$
\begin{aligned}
\int_{-\pi}^{\pi} \varphi\left(R e^{i \theta}\right) V(\theta) d \theta & =\frac{2^{\beta+\gamma}}{\Gamma(\beta+\gamma)} \int_{0}^{1}\left(\log \frac{1}{\rho}\right)^{\beta+\gamma-1} \frac{d \rho}{\rho} \int_{-\pi}^{\pi} \vartheta^{\beta} \varphi\left(\rho e^{i \theta}\right) \vartheta^{\gamma} \xi\left(R \rho e^{-i \theta}\right) d \theta \\
& =\frac{2^{\beta+\gamma}}{\Gamma(\beta+\gamma)} \int_{-\pi}^{\pi} d \theta \int_{0}^{1}\left(\log \frac{1}{\rho}\right)^{\beta+\gamma-1} \vartheta^{\beta} \varphi\left(\rho e^{i \theta}\right) \vartheta \gamma \xi\left(R \rho e^{-i \theta}\right) \frac{d \rho}{\rho} .
\end{aligned}
$$

By Hölder's inequality with indices $k, k^{\prime}$, the absolute value of the inner integral on the right of (8.4) does not exceed $\mathscr{G}_{k, \beta}(\theta) \mathscr{C}_{k^{\prime}, \gamma}(-\theta)$, and therefore, by Hölder's inequality with indices $p, p^{\prime}$.

$$
\begin{aligned}
\left|\frac{1}{2 \pi} \int_{-\pi}^{\pi} \varphi\left(R e^{i \theta}\right) V(\theta) d \theta\right| & \leqq \frac{2^{\beta+\gamma}}{2 \pi \Gamma(\beta+\gamma)} \int_{-\pi}^{\pi} \mathscr{G}_{k, \beta}(\theta) \mathscr{C}_{k^{\prime}, \gamma}(-\theta) d \theta \\
& \leqq \frac{2^{\beta+\gamma}}{\Gamma(\beta+\gamma)} \mathscr{C}_{p}\left(\mathscr{G}_{k, \beta}\right) \mathscr{C}_{p^{\prime}}\left(\mathscr{H}_{k^{\prime}, \gamma}\right) .
\end{aligned}
$$


Taking $\gamma=1$ (say), we obtain from (8.5) and (8.3) the inequality (8.2), and this completes the proof.

When $p=+\infty$, the inequality (5.4) is false for all $\beta>0$. To prove this, we take $\varphi(z)=\sum_{n=2}^{\infty}(n \log n)^{-1} z^{n}$, so that $\varphi$ is unbounded in $\Delta$. Then for $\beta>0$ we have

$$
\left|\vartheta^{\beta} \varphi\left(\rho e^{i \theta}\right)\right| \leqq \sum_{n=2}^{\infty} n^{\beta-1}(\log n)^{-1} \rho^{n} \leqq A(\beta) \rho^{2}(1-\rho)^{-\beta}\left(\log \frac{e}{1-\rho}\right)^{-1},
$$

whence $\mathscr{G}_{k, \beta}(\theta) \leqq A(k, \beta)$ for all $\theta$ (since $k>1$ ), and this proves the statement.

We note in passing that the results for the function $g_{k, \beta}$ defined in (5.5) corresponding to Theorems 3 and 4 are now immediate consequences of (5.6) and (5.7). When $k>1$ we have also an inequality for $g_{k, \beta}$ corresponding to $(5.2)$, but we postpone the proof of this until $\S 16$.

9. It is probable that the inequality of Theorem 4 holds for $p>0,0<k \leqq 2, \beta>0$. We are unable to prove this in full generality, but we can deal with the case $0<k \leqq 1$ for certain values of $\beta .^{3}$ In contrast to Theorem 4 , the case $p=+\infty$ is true here.

THEOREM 5. If $\varphi(0)=0$, and either (i) $0<p \leqq+\infty, 0<k \leqq 1$, $\beta \geqq 1 / k$, or (ii) $0<p=k \leqq 1, \beta>0$, then

$$
\mathscr{C}_{p}(\varphi)=A(k, p, \beta) \mathscr{C l}_{p}\left(\mathscr{G}_{k, \beta}\right) \text {. }
$$

We consider first the case where $\varphi$ is regular in the closed disc $\bar{\Delta}$, and we show that in this case the inequality (9.1) holds for $0<p \leqq+\infty, 0<k \leqq 1, \beta>0$; the limitations on $p$ and $\beta$ in (i) and (ii) arise only in the reduction of the general case to this special one.

Suppose then that $\phi$ is regular in $\bar{\Delta}$ and that $0<p \leqq+\infty$, $0<k \leqq 1, \beta>0$. It is enough to show that

$$
M_{p}(\varphi ; 1) \leqq A(k, p, \beta) \mathscr{C l}_{p}\left(\mathscr{G}_{k, \beta}\right) .
$$

Since $\varphi$ is regular in $\bar{\Delta}$, the formulae (3.7) give

$$
\varphi\left(e^{i \theta}\right)=\frac{1}{\Gamma(\beta)} \int_{0}^{1}\left(\log \frac{1}{\rho}\right)^{\beta-1} \vartheta^{\beta} \varphi\left(\rho e^{i \theta}\right) \frac{d \rho}{\rho},
$$

and therefore

$$
\left|\varphi\left(e^{i \theta}\right)\right| \leqq \frac{1}{\Gamma(\beta)} \int_{0}^{1}\left(\log \frac{1}{\rho}\right)^{\beta-1}\left|\vartheta^{\beta} \varphi\left(\rho e^{i \theta}\right)\right| \frac{d \rho}{\rho} .
$$

${ }^{3}$ A partial result for $0<p \leqq 1, k=2, \beta=1$ is proved in [2]. 
This trivially implies (9.2) for $k=1$, so that we may suppose $k<1$. Let $\Phi$ be defined as in Theorem 1 , with $\eta=1 / 2$ (say). Then

$$
\left|\vartheta^{\beta} \varphi\left(\rho e^{i \theta}\right)\right| \leqq A(\beta) \rho(1-\rho)^{-\beta} \Phi(\theta) \leqq A(\beta)(\log 1 / \rho)^{-\beta} \Phi(\theta),
$$

whence, by $(9.3)$,

$$
\begin{aligned}
\left|\varphi\left(e^{i \theta}\right)\right| & \leqq A(k, \beta) \Phi^{1-k}(\theta) \int_{0}^{1}(\log 1 / \rho)^{k_{\beta} \beta-1}\left|\vartheta^{\beta} \varphi\left(\rho e^{i \theta}\right)\right|^{k} \rho^{-1} d \rho \\
& =A(k, \beta) \Phi^{1-k}(\theta) \mathscr{G}_{k, \beta}^{k}(\theta) .
\end{aligned}
$$

If $p<+\infty$, then (9.4) gives

$$
M_{p}(\varphi ; 1) \leqq A(k, p, \beta)\left\{\int_{-\pi}^{\pi} \Phi^{(1-k) p}(\theta) \mathscr{G}_{k, \beta}^{k p}(\theta) d \theta\right\}^{1 / p} .
$$

Applying Hölder's inequality with indices $1 /(1-k), 1 / k$, and then Theorem E, we obtain

$$
\begin{aligned}
M_{p}(\varphi ; 1) & \leqq A(k, p, \beta) \mathscr{C}_{p}^{(1-k)}(\Phi) \mathscr{C}_{p}^{k}\left(\mathscr{G}_{k, \beta}\right) \\
& \leqq A(k, p, \beta) M_{p}^{1-k}(\varphi ; 1) \mathscr{C}_{p}^{k}\left(\mathscr{G}_{k, \beta}\right),
\end{aligned}
$$

and since $M_{p}(\varphi ; 1)$ is finite, this implies (9.2). If $p=+\infty$, then (9.5) follows immediately from (9.4), and again we obtain (9.2).

Suppose now that $\varphi$ is regular in $\Delta$, and let $0<R<1$. Applying the special case to the function $z m \varphi(R z)$, we get

$$
M_{p}^{p}(\varphi ; R) \leqq A(k, p, \beta) \int_{-\pi}^{\pi} d \theta\left\{\int_{0}^{1}\left(\log \frac{1}{\rho}\right)^{k \beta-1}\left|\vartheta^{\beta} \phi\left(R \rho e^{i \theta}\right)\right|^{k} \frac{d \rho}{\rho}\right\}^{p / k} .
$$

If $\beta \geqq 1 / k$, then

$$
\begin{array}{r}
\int_{0}^{1}\left(\log \frac{1}{\rho}\right)^{k \beta-1}\left|\vartheta^{\beta} \varphi\left(R \rho e^{i \theta}\right)\right|^{k} \frac{d \rho}{\rho}=\int_{0}^{R}\left(\log \frac{R}{\sigma}\right)^{k \beta-1}\left|\vartheta^{\beta} \varphi\left(\sigma e^{i \theta}\right)\right|^{k} \frac{d \sigma}{\sigma} \\
\leqq \int_{0}^{R}\left(\log \frac{1}{\sigma}\right)^{k \beta-1}\left|\vartheta^{\beta} \varphi\left(\sigma e^{i \theta}\right)\right|^{k} \frac{d \sigma}{\sigma} \leqq \mathscr{G}_{k, \beta}^{k}(\theta) .
\end{array}
$$

Hence

$$
M_{p}^{p}(\varphi ; R) \leqq A(k, p, \beta) \mathscr{C l}_{p}^{p}\left(\mathscr{G}_{k, \beta}\right),
$$

and this implies (9.1).

If $k=p, \beta>0$, then (9.6) gives

$$
M_{k}^{k}(\varphi ; R) \leqq A(k, \beta) \int_{0}^{1}\left(\log \frac{1}{\rho}\right)^{k \beta-1} \frac{d \rho}{\rho} \int_{-\pi}^{\pi}\left|\vartheta^{\beta} \varphi\left(R \rho e^{i \theta}\right)\right|^{k} d \theta
$$

Since the inner integral on the right increases with $R$, we may replace $R$ on the right by 1 , and this again implies (9.1).

We note explicitly the cases $p=k$ of Theorems 4 and 5, viz. 
THEOREM 6. If $\varphi(0)=0$, and $0<k \leqq 2, \beta>0$, then

$$
\mathscr{C l}_{k}(\varphi) \leqq A(k, \beta)\left\{\int_{-\pi}^{\pi} \int_{0}^{1}\left(\log \frac{1}{\rho}\right)^{k, \beta-1}\left|\vartheta^{3} \varphi\left(\rho e^{i \theta}\right)\right|^{k} \rho^{-1} d \rho d \theta\right\}^{1 / k} .
$$

10. A theorem on the means $M_{p}(\varphi ; \rho)$. We prove next and let

Theorem 7. Let $\varphi(0)=0$, let $0<p<q \leqq+\infty, \alpha=1 / p-1 / q, k \geqq p$,

$$
J=\left\{\int_{0}^{1}\left(\log \frac{1}{\rho}\right)^{k \alpha-1} M_{q}^{k}(\varphi ; \rho) \frac{d \rho}{\rho}\right\}^{1 / k}
$$

Then

$$
J \leqq A(k, p, q) \mathscr{C}_{p}(\varphi)
$$

This is equivalent to a result of Hardy and Littlewood [12, Th. $31 ; 17, \mathrm{Th} .11]^{4}$. The theorem can be proved in various ways, and we give here a variant of the proof in [17] which makes the least demands on the theory of the $H^{p}$ classes.

Suppose first that $p=2$, so that $\alpha=1 / 2-1 / q$, and let $C=\mathscr{C l}_{2}(\varphi)$. Then

$$
\begin{aligned}
M(\varphi ; \rho) & \leqq \sum_{n=1}^{\infty}\left|c_{n}\right| \rho^{n} \\
& \leqq\left(\sum_{n=1}^{\infty}\left|c_{n}\right|^{2} \rho^{n}\right)^{1 / 2}\left(\sum_{n=1}^{\infty} \rho^{n}\right)^{1 / 2} \leqq C\left(\frac{\rho}{1-\rho}\right)^{1 / 2} \leqq C\left(\log \frac{1}{\rho}\right)^{-1 / 2},
\end{aligned}
$$

and therefore for $2 \leqq q \leqq+\infty$

$$
M_{q}(\varphi ; \rho) \leqq M^{1-2 / q}(\varphi ; \rho) M_{2}^{2 / q}(\varphi ; \rho) \leqq C(\log 1 / \rho)^{-\alpha} .
$$

Hence

$$
J^{k} \leqq C^{k-2} \int_{0}^{1}\left(\log \frac{1}{\rho}\right)^{2 \alpha-1} M_{q}^{2}(\varphi ; \rho) \frac{d \rho}{\rho}
$$

Next, since

$$
\varphi\left(\rho e^{i \theta}\right)=\int_{0}^{\rho} \vartheta^{1} \varphi\left(\sigma e^{i \theta}\right) \frac{d \sigma}{\sigma},
$$

4 The equivalence follows by the argument of [17, Lemma $\gamma]$. Hardy and Littlewood use a factor $(1-\rho)^{k \alpha-1}$ in place of the logarithmic factor above; the form given here is more convenient for our applications.

It should be noted that there is a misprint in the statement of the result in [17]; the $C$ on the right of (11.2) on p. 236 should be $C^{l}$. 
Minkowski's inequality gives

$$
M_{q}(\varphi ; \rho) \leqq \int_{0}^{\rho} M_{q}\left(\vartheta^{1} \varphi ; \sigma\right) \frac{d \sigma}{\sigma}
$$

(the case $q=+\infty$ being included), and hence, by (10.3) and the case $l=k=2, \delta=1$ of Lemma 1 ,

$$
\begin{aligned}
J^{k} & \leqq A(k, \alpha) C^{k-2} \int_{0}^{1}\left(\log \frac{1}{\rho}\right)^{2 \alpha+1} M_{q}^{2}\left(\vartheta^{1} \varphi ; \rho\right) \frac{d \rho}{\rho} \\
& =A(k, \alpha) C^{k-2} \int_{0}^{1}\left(\log \frac{1}{\rho}\right)^{2 \alpha+1} M_{q}^{2}\left(\vartheta^{1} \varphi ; \rho^{2}\right) \frac{d \rho}{\rho} .
\end{aligned}
$$

By (10.2) applied to the function $z m \rightarrow \vartheta^{1} \varphi(\rho z)$,

$$
M_{q}^{2}\left(\vartheta^{1} \varphi ; \rho^{2}\right) \leqq(\log 1 / \rho)^{-2 \alpha} M_{2}^{2}\left(\vartheta^{1} \varphi ; \rho\right),
$$

and hence, by (10.4) and (3.7),

$$
\begin{aligned}
J^{k} & \leqq A(k, q) C^{k-2} \int_{0}^{1}\left(\log \frac{1}{\rho}\right) M_{2}^{2}\left(\vartheta^{1} \varphi ; \rho\right) \frac{d \rho}{\rho} \\
& =A(k, q) C^{k-2} \int_{0}^{1}\left(\log \frac{1}{\rho}\right)\left(\sum_{n=1}^{\infty} n^{2}\left|c_{n}\right|^{2} \rho^{2 n}\right) \frac{d \rho}{\rho} \\
& =A(k, q) C^{k-2} \sum_{n=1}^{\infty}\left|c_{n}\right|^{2}=A(k, q) C^{k},
\end{aligned}
$$

and this is (10.1) with $p=2$.

Suppose next that $p \neq 2$. In this case it is enough to prove that if $\psi$ is regular in $\Delta$, and $0<p<q \leqq+\infty, \alpha=1 / p-1 / q, k=p$, then

$$
\left\{\int_{0}^{1}\left(\log \frac{1}{\rho}\right)^{k \alpha-1} M_{q}^{k}(\psi ; \rho) \rho^{k-1} d \rho\right\}^{1 / k} \leqq A(k, p, q) \mathscr{C}_{p}(\psi),
$$

for the inequality (10.1) follows from this with $\psi(z)=z^{-1} \varphi(z)$. Further, by Theorem C, it is enough to prove (10.5) when $\psi$ has no zeros in $\Delta$.

Let $\psi$ be such a function, let $\chi=\psi^{p / 2}, s=2 q / p, l=2 k / p, \beta=$ $1 / 2-1 / s$. Then $s>2, l \geqq 2, l \beta=k \alpha$, and $\mathscr{C}_{p}^{p}(\psi)=\mathscr{L}_{2}^{2}(\chi)$, so that for this $\psi$ the inequality (10.5) is equivalent to

$$
\left\{\int_{0}^{1}\left(\log \frac{1}{\rho}\right)^{l \beta-1} M_{s}^{l}(\chi ; \rho) \rho^{k-1} d \rho\right\}^{1 / l} \leqq A(k, p, q) \mathscr{C l}_{2}(\chi) .
$$

But, by the case $p=2$ of (10.1) applied to $\varphi(z)=z \chi(z)$,

$$
\left\{\int_{0}^{1}\left(\log \frac{1}{\rho}\right)^{l \beta-1} M_{s}^{l}(\chi ; \rho) \rho^{l-1} d \rho\right\}^{1 / l} \leqq A(k, p, q) \mathscr{C l}_{2}(\chi)
$$


If $k \geqq l$, (10.7) implies (10.6) immediately. If $k<l$, then on putting $\rho^{l}=\sigma^{k}$ in the integral on the left of (10.7), and noting that

$$
M_{s}(\chi ; \rho) \geqq M_{s}(\chi ; \sigma)
$$

(since $\rho=\sigma^{k / l}>\sigma$ ), we see that the left side of (10.6) does not exceed $(l / k)^{\beta}$ times that of (10.7), whence again (10.6) follows, and this completes the proof.

For certain $p$ and $q$ we have a stronger result.

THEOREM 8. Let $\varphi(0)=0$, let $w=\left(w_{n}\right)$ be a sequence of numbers such that $\left|w_{n}\right| \leqq 1$ for all $n$, and let

$$
\varphi_{w}(z)=\sum_{n=1}^{\infty} c_{n} w_{n} z^{n} \quad(z \in A) .
$$

If $0<p<q \leqq+\infty, p \leqq 2 \leqq q, \alpha=1 / p-1 / q, k \geqq p$, then

$$
\left\{\int_{0}^{1}\left(\log \frac{1}{\rho}\right)^{k \alpha-1} M_{q}^{k}\left(\varphi_{w} ; \rho\right) \frac{d \rho}{\rho}\right\}^{1 / k} \leqq A(k, p, q) \mathscr{C l}_{p}(\varphi) \text {. }
$$

If $p=2$, this follows from the trivial inequality $-\mathbb{C}_{2}\left(\varphi_{w}\right) \leqq \mathbb{C}_{2}(\phi)$ and the inequality (10.1) applied to $\varphi_{w}$.

If $p<2$, then by (10.1) with $q=2$ we have

$$
\left\{\int_{0}^{1}\left(\log \frac{1}{\rho}\right)^{k / p-k / 2-1} M_{2}^{k}(\varphi ; \rho) \frac{d \rho}{\rho}\right\}^{1 / k} \leqq A(k, p) \mathscr{C l}_{p}(\varphi) .
$$

Further, by (10.2) applied to $\varphi_{w}$, we have

$$
(\log 1 / \rho)^{1 / 2-1 / q} M_{q}\left(\varphi_{w} ; \rho\right) \leqq M_{2}\left(\varphi_{w} ; \rho\right) \leqq M_{2}(\varphi ; \rho)
$$

for $2 \leqq q \leqq+\infty$, and (10.10) and (10.11) together give (10.9).

Choosing $w$ in Theorem 8 so that $c_{n} w_{n}=\left|c_{n}\right|$ for all $n$, we deduce the following result.

Theorem 8. Corollary. Let $\varphi(0)=0$, and let

$$
\varphi_{*}(z)=\sum_{n=1}^{\infty}\left|c_{n}\right| z^{n} \quad(z \in \Delta) .
$$

If $0<p<q \leqq+\infty, p \leqq 2 \leqq q, \alpha=1 / p-1 / q, k \geqq p$, then

$$
\left\{\int_{0}^{1}\left(\log \frac{1}{\rho}\right)^{k \alpha-1} M_{q}^{k}\left(\varphi_{*} ; \rho\right) \frac{d \rho}{\rho}\right\}^{1 / k} \leqq A(k, p, q) \mathscr{C l}_{p}(\varphi) .
$$

In particular, if $0<p \leqq 2, k \geqq p$, then 


$$
\left\{\int_{0}^{1}\left(\log \frac{1}{\rho}\right)^{k / p-1} \varphi_{*}^{k}(\rho) \frac{d \rho}{\rho}\right\}^{1 / k} \leqq A(k, p) \mathscr{C l}_{p}(\varphi)
$$

and if $0<p \leqq 2$, then

$$
\left\{\int_{0}^{1} \varphi_{*}^{p}(\rho) \frac{d \rho}{\rho}\right\}^{1 / p} \leqq A(p)-\not l{ }_{p}(\varphi)
$$

The inequality (10.14) is equivalent to a theorem of Hardy and Littlewood [8, Th. 15], and $(10.13)$ can be deduced from two results of the same authors [10, Th. 3; 8, Th. 5]. The proofs of these results given by Hardy and Littlewood make use of the inequality

$$
\left\{\sum_{n=1}^{\infty} n^{p-2}\left|c_{n}\right|^{p}\right\}^{1 / p} \leqq A(p) \mathscr{C l}_{p}(\varphi),
$$

where $1<p \leqq 2$, and are a good deal less elementary than the proof above.

It has been shown by Hardy and Littlewood [8] that for $0<p \leqq 1$ the inequality (10.14) implies (10.15), the argument here being relatively simple. We thus obtain effectively a new proof of (10.15) for $0<p \leqq 1$.

It is natural here to ask whether

$$
\mathscr{C}_{p}\left(\varphi_{w}\right) \leqq A(p) \mathscr{K}_{p}(\varphi) \quad(p>0, p \neq 2)
$$

for every sequence $w=\left(w_{n}\right)$ such that $\left|w_{n}\right| \leqq 1$. As might be expected, the answer is negative. If (10.16) were true for $p>2$, then, by Theorem $7,(10.14)$ would hold for $p>2$, and this is known to be false, a counter-example being

$$
\varphi(z)=\sum n^{-1 / 2-\delta} e^{i n \log n} z^{n} \quad(\delta>0)
$$

(Hardy and Littlewood [8, p. 206]). This argument shows also that the inequality

$$
\mathscr{C l}_{p}\left(\varphi_{*}\right) \leqq A(p) \mathscr{C l}_{p}(\varphi)
$$

is false for $p>2$.

To disprove (10.16) for $p<2$, we may take

$$
\varphi(z)=\sum n^{-1 / 2} z^{n}, \quad w_{n}=e^{i n \log n} .
$$

Here $\varphi \in H^{p}$ for $p<2$. On the other hand, $\varphi_{w}$ has nowhere a radial limit, so that $\mathscr{C l}_{p}\left(\varphi_{w}\right)=+\infty$ for all $p$ (see [22, i, p. 186] and [21]).

The question whether (10.17) holds for $p<2$ seems to be open (see [15]). 
11. The Hardy-Littlewood theorem on fractional integrals. The preceding results enable us to give a succinct proof of the HardyLittlewood theorem on fractional integrals $([12,17]$; see also $[22$, ii, p. 140]).

TheOREM 9. If $\varphi(0)=0$, and $q>p>0, \alpha=1 / p-1 / q$, then

$$
\mathscr{C}_{q}\left(\vartheta_{\alpha} \varphi\right) \leqq A(p, q) \sim \mathscr{C}_{p}(\varphi) \text {. }
$$

Suppose first that $p \leqq 2$, and let $k=\min \{q, 2\}$. Then, by Theorem 6 and the case $k=2$ of Theorem 4 , we have

$$
\mathscr{C l}_{q}(\phi) \leqq A(q, \alpha)\left\{\int_{-\pi}^{\pi} d \theta\left\{\int_{0}^{1}\left(\log \frac{1}{\rho}\right)^{k \alpha-1}\left|\vartheta^{\alpha} \phi\left(\rho e^{i \theta}\right)\right|^{k} \frac{d \rho}{\rho}\right\}^{q / k}\right\}^{1 / q} .
$$

Since $\vartheta^{\alpha}\left(\vartheta_{\alpha} \varphi\right)=\varnothing$, applying successively (11.2) with $\varphi$ replaced by $\vartheta_{\alpha} \varphi$, Minkowski's inequality, and Theorem 7 , we obtain

$$
\begin{aligned}
\mathscr{C l}_{q}\left(\vartheta_{\alpha} \varphi\right) & \leqq A(p, q)\left\{\int_{-\pi}^{\pi} d \theta\left\{\int_{0}^{1}\left(\log \frac{1}{\rho}\right)^{k \alpha-1}\left|\varphi\left(\rho e^{i \theta}\right)\right|^{k} \frac{d \rho}{\rho}\right\}^{q / k}\right\}^{1 / q} \\
& \leqq A(p, q)\left\{\int_{0}^{1}\left(\log \frac{1}{\rho}\right)^{k \alpha-1} M_{q}^{k}(\varphi ; \rho) \frac{d \rho}{\rho}\right\}^{1 / k} \\
& \leqq A(p, q) \mathscr{C}_{p}(\varphi)
\end{aligned}
$$

as required.

This leaves only the case $q>p>2$. To deal with this, we can use a simple conjugacy argument which enables us to deduce the required result from the case $1<p<q<2$ already proved. Since the argument is a particular case of one given in $\$ 13$, we omit it here (see [22, ii, p. 141]).

If $0<p \leqq 1$, the result of Theorem 9 continues to hold for $q=+\infty$. To prove this we use the case $k=1, p=+\infty$ of Theorem 5(i) and Theorem 7. We thus obtain

$$
\begin{aligned}
\mathscr{Z}\left(\vartheta_{1 / p} \varphi\right) & \leqq A(p) \sup _{\theta}\left\{\int_{0}^{1}\left(\log \frac{1}{\rho}\right)^{1 / p-1}\left|\varphi\left(\rho e^{i \theta}\right)\right| \frac{d \rho}{\rho}\right\} \\
& \leqq A(p) \int_{0}^{1}\left(\log \frac{1}{\rho}\right)^{1 / p-1} M(\varphi ; \rho) \frac{d \rho}{\rho} \leqq A(p) \sim \mathbb{C}_{p}(\varphi) .
\end{aligned}
$$

This can be strengthened slightly, as can also the case $p \leqq 2 \leqq q$ of Theorem 9 . Let $w=\left(w_{n}\right)$ be a sequence of numbers such that $\left|w_{n}\right| \leqq 1$, and let $\varphi_{w}$ be defined as in (10.8). Since

$$
\vartheta_{\alpha} \varphi_{w}=\vartheta_{1 / p-1 / q} \varphi_{w}=\vartheta_{1 / 2-1 / q}\left(\vartheta_{1 / p-1 / 2} \varphi_{w}\right)
$$

we have 


$$
\mathscr{C}_{q}\left(\vartheta_{\alpha} \varphi_{w}\right) \leqq A(q) \mathscr{M}_{2}\left(\vartheta_{1 / p-1 / 2} \varphi_{w}\right) \leqq A(q) \mathscr{C l}_{2}\left(\vartheta_{1 / p-1 / 2} \varphi\right) \leqq A(p, q) \mathscr{C l}_{p}(\varphi) \text {, }
$$

by a double application of Theorem 9 . In particular, if $\varphi_{*}$ is the majorant of $\varphi$ defined in (10.12), then

$$
\mathscr{C}_{q}\left(\vartheta_{\alpha} \varphi_{*}\right) \leqq A(p, q) \mathscr{M}_{p}(\varphi) \text {. }
$$

It follows from a theorem of Hardy and Littlewood on majorants [11] that (11.4) is stronger than (11.1) when $q$ is an even integer, and it is probably stronger for all $q \geqq 2$.

If $0<p \leqq 1, q=+\infty$, then the argument above can be combined with that of (11.3), and (with $\varphi_{w}=\varphi_{*}$ ) gives the inequality

$$
\sum_{n=1}^{\infty} n^{-1 / p}\left|c_{n}\right| \leqq A(p) \mathscr{M}_{p}(\varphi) \quad(0<p \leqq 1) .
$$

This, however, is weaker than the case $0<p \leqq 1$ of (10.15) (see Hardy and Littlewood [12, p. 421]).

12. Theorem 6 enables us also to give a simplified proof of the following theorem of Hardy and Littlewood [12, Th. 46].

Theorem 10. Let $0<p \leqq+\infty, 0<\alpha<\gamma$, let $\varphi(0)=0$, and let

$$
M_{p}(\varphi ; \rho) \leqq(\log 1 / \rho)^{-\gamma} \quad(0<\rho<1) .
$$

Then

$$
M_{p}\left(\vartheta_{\alpha} \varphi ; \rho\right) \leqq A(p, \alpha, \gamma)(\log 1 / \rho)^{\alpha-\gamma} \quad(0<\rho<1) .
$$

Suppose first that $1 \leqq p \leqq+\infty$. By (3.6),

$$
\vartheta_{\alpha} \varphi\left(\rho e^{i \theta}\right)=\frac{1}{\Gamma(\alpha)} \int_{0}^{\rho}\left(\log \frac{\rho}{\sigma}\right)^{\alpha-1} \varphi\left(\sigma e^{i \theta}\right) \frac{d \sigma}{\sigma},
$$

whence, by Minkowski's inequality,

$$
\begin{aligned}
M_{p}\left(\vartheta_{\alpha} \varphi ; \rho\right) & \leqq \frac{1}{\Gamma(\alpha)} \int_{0}^{\rho}\left(\log \frac{\rho}{\sigma}\right)^{\alpha-1} M_{p}(\varphi ; \sigma) \frac{d \sigma}{\sigma} \\
& \leqq \frac{1}{\Gamma(\alpha)} \int_{0}^{\rho}\left(\log \frac{\rho}{\sigma}\right)^{\alpha-1}\left(\log \frac{1}{\sigma}\right)^{-\gamma} \frac{d \sigma}{\sigma} \\
& =\frac{\Gamma(\gamma-\alpha)}{\Gamma(\gamma)}\left(\log \frac{1}{\rho}\right)^{\alpha-\gamma},
\end{aligned}
$$

the last integral in (12.1) being evaluated by the substitution

$$
1 / y=\log 1 / \sigma, \quad 1 / x=\log 1 / \rho .
$$

Suppose next that $0<p<1$. By Theorem 6 with $k=p, \beta=\alpha$, 
applied to the function $z m \vartheta_{\alpha} \varphi(\rho z)$, we have

$$
\begin{aligned}
M_{p}^{p}\left(\vartheta_{\alpha} \varphi ; \rho\right) & \leqq A(p, \alpha) \int_{0}^{1}\left(\log \frac{1}{t}\right)^{p \alpha-1} M_{p}^{p}(\varphi ; \rho t) \frac{d t}{t} \\
& \leqq A(p, \alpha) \int_{0}^{1}\left(\log \frac{1}{t}\right)^{p \alpha-1}\left(\log \frac{1}{\rho t}\right)^{-p \gamma} \frac{d t}{t} \\
& =A(p, \alpha) \int_{0}^{\rho}\left(\log \frac{\rho}{\sigma}\right)^{p a-1}\left(\log \frac{1}{\sigma}\right)^{-p \gamma} \frac{d \sigma}{\sigma} \\
& =A(p, \alpha, \gamma)\left(\log \frac{1}{\rho}\right)^{p(\alpha-\gamma)}
\end{aligned}
$$

(by the same substitution as before), and this completes the proof.

Combining Theorem 10 with Theorem 1, Corollary 2, we obtain the following result (cf. [12, Th. 46]).

THEOREM 11. Let $0<p \leqq+\infty, \gamma>0, \gamma>\alpha$, let $\varphi(0)=0$, and let

$$
M_{p}(\varphi ; \rho) \leqq(\log 1 / \rho)^{-r} \quad(0<\rho<1)
$$

Then

$$
M_{p}\left(\vartheta_{\alpha} \varphi ; \rho\right) \leqq A(p, \alpha, \gamma)(\log 1 / \rho)^{\alpha-\gamma} \quad(0<\rho<1) .
$$

13. The convolution series of two power series. We suppose throughout this section that $\varphi, \psi$ are regular in $\Delta$, and that

$$
\varphi(z)=\sum_{n=1}^{\infty} c_{n} z^{n}, \quad \psi(z)=\sum_{n=1}^{\infty} d_{n} z^{n}, \quad \chi(z)=\sum_{n=1}^{\infty} c_{n} d_{n} z^{n} .
$$

It is easily verified that $\chi$ is regular in $\Delta$, and that

$$
\chi\left(\rho^{2} e^{i \theta}\right)=\frac{1}{2 \pi} \int_{-\pi}^{\pi} \varphi\left(\rho e^{i \theta-i t}\right) \psi\left(\rho e^{i t}\right) d t .
$$

It follows immediately from Theorem $\mathrm{A}$ that if

$$
p \geqq 1, \quad q \geqq 1, \quad \frac{1}{r}=\frac{1}{p}+\frac{1}{q}-1 \geqq 0
$$

(so that $\max \{p, q\} \leqq r \leqq+\infty$ ), and $\varphi \in H^{p}$, is $\in H^{q}$, then $\chi \in H^{r}$.

Hardy and Littlewood $[16,17]$ have given generalizations of this result in which the condition that $\psi \in H^{q}$ is replaced by the condition that

$$
M_{q}\left(\psi^{\prime} ; \rho\right) \leqq K(1-\rho)^{k-1}
$$

for some $k$. If $k=0$, then (13.1) is weaker than the condition that 
$\psi \in H^{q}$ (cf. Theorem 1, Corollary 1); however, the conclusion that $\chi \in H^{r}$ remains valid. If $0<k<1$, then (13.1) is equivalent to the condition that $\psi \in \operatorname{Lip}(k, q)$ (Hardy and Littlewood [11, Th. 3]), and is stronger than the condition that $\psi \in H^{q}$. In this case the conclusion that $\chi \in H^{r}$ remains valid when $\varphi \in H^{s}$ for some $s<p$.

In this section we generalize these theorems by replacing (13.1) by a similar condition involving $M_{q}\left(\vartheta^{3} \psi ; \rho\right)$, where $\beta>0$. Such results were stated by Hardy and Littlewood [16] for the case where $\beta$ is a positive integer $m$, but no proof for $m>1$ has been published. We find in fact that there are three distinct theorems ${ }^{5}$.

THEOREM 12. Suppose that

$$
p \geqq 1, \quad q \geqq 1, \quad \frac{1}{r}=\frac{1}{p}+\frac{1}{q}-1>0, \quad p \leqq 2 \leqq r, \quad \beta \geqq 0,
$$

that $\varphi \in H^{p}$, and that

$$
M_{q}\left(\vartheta^{\beta} \psi ; \rho\right) \leqq K(\log 1 / \rho)^{-\beta} .
$$

Then

$$
\mathscr{K}_{r}(\chi) \leqq K A(p, q, \beta) \mathscr{C}_{p}(\varphi)
$$

In the remaining two theorems we regard $p, q, \lambda, \beta$ as given, and define $r, s$ in terms of them.

TheOREM 13. Suppose that

$$
\begin{gathered}
p \geqq 1, \quad q \geqq 1, \quad \frac{1}{r}=\frac{1}{p}+\frac{1}{q}-1 \geqq 0, \\
0 \leqq \lambda<\beta, \quad \frac{1}{s}=\frac{1}{p}+\beta-\lambda
\end{gathered}
$$

(so that $0<s<p$ ), that $\varphi \in H^{s}$, and that

$$
M_{q}\left(\vartheta^{\beta} \psi ; \rho\right) \leqq K(\log 1 / \rho)^{-\lambda} \text {. }
$$

If $r<+\infty$ (so that $1 / p+1 / q>1$ ), then

$$
\mathscr{C l}_{r}(\chi) \leqq K A(p, q, \beta, \lambda) \sim \mathscr{C}_{s}(\varphi) \text {. }
$$

If $r=+\infty$ (so that $1 / p+1 / q=1$ ) and $s \leqq 1$, then $\chi$ is continuous in $\bar{A}$, and for each $\theta$

5 The case $\beta \neq 1$ of Theorem 12 can be reduced to the case $\beta=1$ by means of Theorem 1, Corollary 2 and Theorem 10. Similarly, Theorems 13 and 14 can be reduced to the case of integral $\beta$ by means of Theorem 10. However. our proofs of Theorems 12-14 apply equally to integral and nonintegral $\beta$. 


$$
\left|\chi\left(e^{i \theta}\right)\right| \leqq \frac{1}{\Gamma(\beta)} \int_{0}^{1}\left(\log \frac{1}{\rho}\right)^{\beta-1}\left|\vartheta^{\beta} \chi\left(\rho e^{i \theta}\right)\right| \frac{d \rho}{\rho} \leqq K A(p, q, \beta, \lambda) \mathscr{C l}_{s}(\varphi) .
$$

THeOREM 14. Suppose that the hypotheses of Theorem 13 hold, and that in addition $s \leqq 2 \leqq p$. Suppose also that $w=\left(w_{n}\right)$ is a sequence of numbers such that $\left|w_{n}\right| \leqq 1$ for all $n$, and let

$$
\chi_{w}(z)=\sum_{n=1}^{\infty} c_{n} d_{n} w_{n} z^{n} \quad(z \in \Delta) .
$$

If $r<+\infty$ (so that $1 / p+1 / q>1)$, then

$$
\mathscr{C}_{r}\left(\chi_{w}\right) \leqq K A(p, q, \beta, \lambda) \mathscr{C}_{s}(\varphi),
$$

and, in particular, if

$$
\chi_{*}(z)=\sum_{n=1}^{\infty}\left|c_{n} d_{n}\right| z^{n} \quad(z \in \Delta),
$$

then

$$
\mathscr{C}_{r}\left(\chi_{*}\right) \leqq K A(p, q, \beta, \lambda) \mathscr{C l}_{s}(\varphi) .
$$

If $r=+\infty$ (so that $1 / p+1 / q=1$ ) and $s \leqq 1$, then

$$
\sum_{n=1}^{\infty}\left|c_{n} d_{n}\right| \leqq K A(p, q, \beta, \lambda) \mathscr{C}_{s}(\varphi) \text {. }
$$

Proofs of the cases $\beta=1, r<+\infty$ of Theorems 12 and 13 are given by Hardy and Littlewood in [16, 17]. They have also proved in [13, 14] the cases $\beta=1$ of the inequalities (13.3) and (13.5). The proofs of Theorems 12 and 13 given here are similar in principle to those of the cases $\beta=1$ in [17], but we have made some simplications.

In the proofs of Theorems 12-14 we may assume that $K=1$, and in Theorem 12 we may assume $\beta>0$. We write $B$ for a constant depending on some or all of the parameters concerned, and we suppose that

$$
p \geqq 1, \quad q \geqq 1, \quad \frac{1}{r}=\frac{1}{p}+\frac{1}{q}-1 \geqq 0, \quad 0 \leqq \lambda \leqq \beta, \quad \beta>0,
$$

and

$$
M_{q}\left(\vartheta^{\beta} \psi ; \rho\right) \leqq(\log 1 / \rho)^{-\lambda}
$$

We observe now that, by Parseval's theorem, for any real $\gamma$ we have

$$
\vartheta^{\beta+\gamma} \chi\left(\rho^{2} e^{i \theta}\right)=\frac{1}{2 \pi} \int_{-\pi}^{\pi} \vartheta^{\gamma} \varphi\left(\rho e^{i \theta-i t}\right) \vartheta^{\beta} \psi\left(\rho e^{i t}\right) d t
$$


and hence, by Theorem $\mathrm{A}$,

$$
\begin{aligned}
M_{r}\left(\vartheta^{\beta+\gamma} \chi ; \rho^{2}\right) & \leqq M_{p}\left(\vartheta^{r} \varphi ; \rho\right) M_{q}\left(\vartheta^{\beta} \psi ; \rho\right) \\
& \leqq(\log 1 / \rho)^{-\lambda} M_{p}\left(\vartheta^{r} \varphi ; \rho\right) .
\end{aligned}
$$

Consider first the proof of Theorem 12. Here $p \leqq 2 \leqq r<+\infty$ and $\lambda=\beta$, and we choose $\gamma$ in (13.6) to be a fixed positive number (e.g. $\gamma=1$ ). Applying successively Theorem 4 with $k=2$ and $\beta$ replaced by $\beta+\gamma$, Minkowski's inequality, the inequality (13.6), Minkowski's inequality again, and Theorem 3 , we obtain

$$
\begin{aligned}
\mathscr{C}_{r}(\chi) & \leqq B\left\{\int_{-\pi}^{\pi} d \theta\left\{\int_{0}^{1}\left(\log \frac{1}{\rho}\right)^{2 \beta+2 \gamma-1}\left|\vartheta^{\beta+\gamma} \chi\left(\rho e^{i \theta}\right)\right|^{2} \frac{d \rho}{\rho}\right\}^{r / 2}\right\}^{1 / r} \\
& \leqq B\left\{\int_{0}^{1}\left(\log \frac{1}{\rho}\right)^{2 \beta+2 \gamma-1} M_{r}^{2}\left(\vartheta^{\beta+\gamma} \chi ; \rho\right) \frac{d \rho}{\rho}\right\}^{1 / 2} \\
& =B\left\{\int_{0}^{1}\left(\log \frac{1}{\rho}\right)^{2, \beta+2 \gamma-1} M_{r}^{2}\left(\vartheta^{\beta+\gamma} \chi ; \rho^{2}\right) \frac{d \rho}{\rho}\right\}^{1 / 2} \\
& \leqq B\left\{\int_{0}^{1}\left(\log \frac{1}{\rho}\right)^{2 \gamma+1} M_{p}^{2}\left(\vartheta^{\gamma} \varphi ; \rho\right) \frac{d \rho}{\rho}\right\}^{1 / 2} \\
& \leqq B\left\{\int_{-\pi}^{\pi} d \theta\left\{\int_{0}^{1}\left(\log \frac{1}{\rho}\right)^{2 \gamma+1}\left|\vartheta^{\gamma} \varphi\left(\rho e^{i \theta}\right)\right|^{2} \frac{d \rho}{\rho}\right\}^{p / 2}\right\}^{1 / p} \\
& \leqq B \mathbb{C l}_{p}(\varphi),
\end{aligned}
$$

and this is the required result.

We prove next the case $s \leqq 2, r<+\infty$ of Theorem 13. Let $k=\min \{r, 2\}$. Then, by Theorem 6 and the case $k=2$ of Theorem 4,

$$
\mathscr{C l}_{r}(\chi)=B\left\{\int_{-\pi}^{\pi} d \theta\left\{\int_{0}^{1}\left(\log \frac{1}{\rho}\right)^{k \beta-1}\left|\vartheta^{3} \chi\left(\rho e^{i \theta}\right)\right|^{k} \frac{d \rho}{\rho}\right\}^{r / k}\right\}^{1 / r} .
$$

Applying successively Minkowski's inequality, the inequality (13.6) with $\gamma=0$, and Theorem 7 , we obtain

$$
\begin{aligned}
\mathscr{C l}_{r}(\chi) & \leqq B\left\{\int_{0}^{1}\left(\log \frac{1}{\rho}\right)^{k \beta-1} M_{r}^{k}\left(\vartheta^{\beta} \chi ; \rho\right) \frac{d \rho}{\rho}\right\}^{1 / k} \\
& =B\left\{\int_{0}^{1}\left(\log \frac{1}{\rho}\right)^{k \beta-1} M_{r}^{k}\left(\vartheta^{\beta} \chi ; \rho^{2}\right) \frac{d \rho}{\rho}\right\}^{1 / k} \\
& \leqq B\left\{\int_{0}^{1}\left(\log \frac{1}{\rho}\right)^{k \beta-k \lambda-1} M_{p}^{k}(\rho ; \rho) \frac{d \rho}{\rho}\right\}^{1 / k} \\
& \leqq B \mathscr{C}_{s}(\varphi)
\end{aligned}
$$

since $0<s<p, s \leqq k, \beta-\lambda=1 / s-1 / p$. This proves the appropriate part of Theorem 13. Similarly, by applying (13.7) to $\chi_{w}$ and using Theorem 8 in place of Theorem 7, we obtain the case $r<+\infty$ of Theorem 14. 
To prove the case $s \leqq 1, r=+\infty$ of Theorem 13 , we note that if $0 \leqq R<1$, then

$$
\chi\left(R e^{i \theta}\right)=\frac{1}{\Gamma(\beta)} \int_{0}^{1}\left(\log \frac{1}{\rho}\right)^{\beta-1} \vartheta^{\beta} \chi\left(R \rho e^{i \theta}\right) \frac{d \rho}{\rho} .
$$

Let $0<\delta<1$. Then, by the increasing property of $M$ and the inequality (13.6) with $\gamma=0$,

$$
\begin{aligned}
& \sup _{R, \theta}\left|\int_{\delta}^{1}\left(\log \frac{1}{\rho}\right)^{\beta-1} \vartheta^{\beta} \chi\left(R \rho e^{i \theta}\right) \frac{d \rho}{\rho}\right| \\
& \leqq \int_{\delta}^{1}\left(\log \frac{1}{\rho}\right)^{\beta-1} M\left(\vartheta^{\beta} \chi ; \rho\right) \frac{d \rho}{\rho} \\
&=2^{\beta} \int_{\sqrt{ } \delta}^{1}\left(\log \frac{1}{\rho}\right)^{\beta-1} M\left(\vartheta^{\beta} \chi ; \rho^{2}\right) \frac{d \rho}{\rho} \\
& \leqq B \int_{\sqrt{ } \delta}^{1}\left(\log \frac{1}{\rho}\right)^{\beta-\lambda-1} M_{p}(\varphi ; \rho) \frac{d \rho}{\rho} .
\end{aligned}
$$

Further, by Theorem 7 ,

$$
\int_{0}^{1}\left(\log \frac{1}{\rho}\right)^{\beta-\lambda-1} M_{p}(\varphi ; \rho) \frac{d \rho}{\rho} \leqq B \mathscr{C}_{s}(\varphi) .
$$

It follows that the integral on the right of (13.8) is convergent, uniformly in $(R, \theta)$, and this implies that $\chi$ is continuous in $\bar{A}$, and that (13.3) holds. A similar argument, using Theorem 8 in place of Theorem 7, gives the corresponding case of Theorem 14 .

There remains the case $s>2$ of Theorem 13, which is deduced by a conjugacy argument from the case already proved. The argument here is identical to that used by Hardy and Littlewood in their proof for the case $\beta=1$, but since the proof is short, we give it for the sake of completeness.

Let $s>2$, so that also $r \geqq p>s>2$. As in the proof of Theorem 4 , it is enough to prove that if $V$ is a trigonometric polynomial satisfying $\mathscr{C}_{r^{\prime}}(V)=1$, then for $0<R<1$

$$
\left|\frac{1}{2 \pi} \int_{-\pi}^{\pi} \chi\left(R^{2} e^{i \theta}\right) V(\theta) d \theta\right| \leqq B \mathscr{C}_{s}(\varphi) .
$$

Let $V(\theta)=\sum_{n=-N}^{N} \kappa_{n} e^{n i \theta}$, and let $\xi(z)=\sum_{n=1}^{N} \kappa_{-n} z^{n}$. Then

$$
\begin{aligned}
\frac{1}{2 \pi} \int_{-\pi}^{\pi} \chi\left(R^{2} e^{i \theta}\right) V(\theta) d \theta & =\frac{1}{4 \pi^{2}} \int_{-\pi}^{\pi} V(\theta) d \theta \int_{-\pi}^{\pi} \varphi\left(R e^{i t}\right) \psi\left(R e^{i \theta-i t}\right) d t \\
& =\frac{1}{2 \pi} \int_{-\pi}^{\pi} \varphi\left(R e^{i t}\right) \zeta\left(R e^{-i t}\right) d t,
\end{aligned}
$$

where 


$$
\zeta\left(R e^{i t}\right)=\frac{1}{2 \pi} \int_{-\pi}^{\pi} V(\theta) \psi\left(R e^{i \theta+i t}\right) d t=\sum_{n=1}^{N} \kappa_{-n} d_{n} R^{n} e^{n i t}
$$

Hence $\xi, \psi, \zeta$ are related as $\varphi, \psi, \chi$ are related in the main theorem. Since also $1<r^{\prime}<s^{\prime}<2, q<s^{\prime}$ and

$$
\frac{1}{r^{\prime}}-\frac{1}{s^{\prime}}=\frac{1}{s}-\frac{1}{r}
$$

we may apply the case of Theorem 13 already proved to $\xi, \psi$, $\zeta$, with $r^{\prime}, s^{\prime}$ in place of $s, r$. Using also Theorem $\mathrm{G}$, we thus obtain

$$
\mathscr{C}_{s^{\prime}}(\zeta) \leqq B \mathscr{M}_{r^{\prime}}(\xi) \leqq B \mathscr{M}_{r^{\prime}}(V)=B
$$

Applying Hölder's inequality with indices $s, s^{\prime}$ to the integral on the right of (13.10), and using (13.11), we obtain (13.9), and this completes the proof.

14. An alternative definition of fractional integral and derivative. An alternative definition of fractional integral which has been used by a number of authors is as follows. As before, let $\varphi$ be regular in $\Delta$, and let

$$
\varphi(z)=\sum_{n=0}^{\infty} c_{n} z^{n} \quad(z \in \Delta) .
$$

Then for any $\alpha \geqq 0$ we define the fractional integral $D_{\alpha} \varphi$ of $\varphi$ of order $\alpha$ by

$$
D_{\alpha} \varphi(z)=z^{\alpha} \sum_{n=0}^{\infty} \frac{\Gamma(n+1)}{\Gamma(n+1+\alpha)} c_{n} z^{n}=\sum_{n=0}^{\infty} \frac{\Gamma(n+1)}{\Gamma(n+1+\alpha)} c_{n} z^{n+\alpha},
$$

where $z^{\alpha}$ has its principal value, i.e.

$$
z^{\alpha}=\exp (\alpha(\log |z|+i \arg z)), \quad-\pi<\arg z \leqq \pi .
$$

This definition is also due to Hadamard [7]. By term-by-term integration, we have

$$
D_{\alpha} \varphi\left(\rho e^{i \theta}\right)=\frac{e^{\alpha i \theta}}{\Gamma(\alpha)} \int_{0}^{\rho}(\rho-\sigma)^{\alpha-1} \varphi\left(\sigma e^{i \theta}\right) d \sigma
$$

where $e^{\alpha i \theta}$ has its principal value.

The definition of the fractional derivative $D^{\beta} \phi$ of order $\beta \geqq 0$ normally associated with the definition (14.1) is that

$$
D^{\beta} \varphi(z)=\left(\frac{d}{d z}\right)^{m} D_{m-\beta} \varphi(z),
$$


where $m=[\beta]+1$ (see Hadamard [7, p. 156]). With this definition we have the series expansion

$$
D^{\beta} \varphi(z)=\sum_{n=0}^{\infty} \frac{\Gamma(n+1)}{\Gamma(n+1-\beta)} c_{n} z^{n-\beta},
$$

where $z^{-\beta}$ has its principal value, and $1 / \Gamma(n+1-\beta)$ is interpreted as 0 when $\beta$ is an integer $\nu \geqq n+1$. When $\beta$ is a positive integer, $D^{\beta} \phi$ is the $\beta$ th derivative of $\varphi$ in the ordinary sense.

The definition (14.2) is satisfactory for $0<\beta<1$, but is less satisfactory for nonintegral $\beta>1$. In particular, the function $D^{\beta} \varphi$ defined above is, for some purposes, too large in the neighborhood of the origin when $\beta>1 .^{6}$

In the sequel we use another definition which avoids these difficulties. For $0 \leqq \beta<1$, we define $D^{\beta} \varphi$ by the series (14.3), and then for $\beta \geqq 1$ we define $D^{\beta} \varphi$ by the relation

$$
D^{\beta} \varphi(z)=D^{\beta-[\beta]}\left(\frac{d}{d z}\right)^{[\beta]} \varphi(z) .
$$

With this definition, we have the series expansion

$$
D^{\beta} \varphi(z)=\sum_{n=[\beta]}^{\infty} \frac{\Gamma(n+1)}{\Gamma(n+1-\beta)} c_{n} z^{n-\beta}
$$

for any $\beta \geqq 0$, where $z^{-\beta}$ has its principal value. Further, if $z=\rho e^{i \theta}$ and $\gamma>\beta \geqq 0$, then

$$
=\sum_{n=[\beta]} D^{\beta} \varphi(z)
$$

where $e^{(\gamma-\beta) i \theta}$ has its principal value. When $\beta$ is a positive integer, $D^{\beta} \varphi$ is the $\beta$ th derivative of $\varphi$ in the ordinary sense, so that in this case the definitions (14.2) and (14.4) agree.

The analogue of Theorem 1 for the derivative $D^{\beta} \phi$ is as follows.

Theorem 15. If $\Phi$ is defined as in Theorem 1 , then for $\beta>0$

$$
\left|D^{\beta} \varphi\left(\rho e^{i \theta}\right)\right| \leqq A(\beta, \eta) \rho^{[\beta]-\beta}(1-\rho)^{-\beta} \Phi(\theta) \quad(0<\rho<1) .
$$

The proof is similar to that of Theorem 1, and we omit it.

15. The function associated with the derivative $D^{\beta}$ correspond-

${ }^{6}$ For instance, with the definition (14.2), the integral on the right of (14.5) below is divergent at the origin for all nonintegral $\gamma>1$. 
ing to the function $\mathscr{G}_{k, \beta}$ is defined by

$$
G_{k, \beta}(\theta)=\left\{\int_{0}^{1}(1-\rho)^{k \beta-1} \rho^{(k-1)(\beta-[\beta])}\left|D^{3} \varphi\left(\rho e^{i \theta}\right)\right|{ }^{k} d \rho\right\}^{1 / k} .
$$

Here it is necessary to insert some power of $\rho$ in the integral to ensure the convergence of the integral at 0 when $\beta-[\beta] \geqq 1 / k$. The particular choice of the power made here enables us to carry over to $G_{k, \beta}$ the argument of Theorem 5, using Theorem 15 in place of Theorem 1. The function $G_{2,1}$ is precisely the Littlewood-Paley $g$-function.

The analogue of Theorem 2 for $G_{k, \beta}$ is more difficult than Theorem 2 itself, and we confine ourselves here to the case $l=k$.

THEOREM 16. If $k \geqq 1$ and $\gamma>\beta>0$, then for each $\theta$

$$
G_{k, \beta}(\theta) \leqq A(k, \beta, \gamma)\left\{\sum_{n=[\beta]}^{[r]-1}\left|c_{n}\right|+G_{k, \gamma}(\theta)\right\} .
$$

The proof of Theorem 16 depends on the following lemmas.

Lemma 2. Let $a>b>0, c>0, y>0$, and let

$$
I=\int_{0}^{1}(x+y)^{-a} x^{b-1}(1-x)^{c-1} d x .
$$

Then

$$
I \leqq A(a, b, c) y^{b-a}(1+y)^{-b} .
$$

Let $B$ denote a constant depending on some or all of $a, b, c$. If $y>1 / 2$ and $0 \leqq x \leqq 1$, then $y \leqq x+y \leqq 3 y$, whence

$$
I \leqq B y^{-a} \int_{0}^{1} x^{b-1}(1-x)^{c-1} d x=B y^{-a},
$$

and this trivially implies (15.2). We may therefore suppose that $0<y \leqq 1 / 2$, and here it is enough to prove that $I \leqq B y^{b-a}$. Write

$$
I=\int_{0}^{y}+\int_{y}^{1 / 2}+\int_{1 / 2}^{1}=I_{1}+I_{2}+I_{3} \text {. }
$$

In $I_{1}, y \leqq x+y \leqq 2 y$ and $(1-x)^{c-1} \leqq B$, whence

$$
I_{1} \leqq B y^{-a} \int_{0}^{y} x^{b-1} d x=B y^{b-a}
$$

In $I_{2}, x \leqq x+y \leqq 2 x$ and $(1-x)^{c-1} \leqq B$, whence

$$
I_{2} \leqq B \int_{y}^{1 / 2} x^{b-a-1} d x \leqq B y^{b-a} .
$$

In $I_{3}, 1 / 2 \leqq x+y \leqq 3 / 2$ and $x^{b-1} \leqq B$, and therefore 


$$
I_{3} \leqq B \int_{1 / 2}^{1}(1-x)^{c-1} d x=B .
$$

Hence $I \leqq B y^{b-a}+B \leqq B y^{b-a}$, as required.

When $a=b+c$, the integral $I$ can be evalued explicity, viz.,

$$
I=\frac{\Gamma(b) \Gamma(c)}{\Gamma(b+c)} y^{b-a}(1+y)^{-b}
$$

(see, for example, [1, i, p. 10, formula (11)]).

We actually use two inequalities derived from Lemma 2 by simple changes of the variable, namely that if $a>b>0, c>0$, then for $0<\rho<1$

$$
\int_{0}^{\rho}(1-\sigma)^{-a}(\rho-\sigma)^{b-1} \sigma^{c-1} d \sigma \leqq A(a, b, c) \rho^{b+c-1}(1-\rho)^{b-a},
$$

and for $0<\sigma<1$

$$
\int_{\sigma}^{1} \rho^{-a}(\rho-\sigma)^{b-1}(1-\rho)^{c-1} d \rho \leqq A(a, b, c)(1-\sigma)^{b+c-1} \sigma^{b-a} .
$$

The next lemma is essentially an extension of the case $l=k$ of Theorem B.

Lemma 3. Let $h$ be a function measurable on the interval ]0,1[, let $h(\rho)>0$ for $0<\rho<1$, and let

$$
H_{\delta}(\rho)=\frac{1}{\Gamma(\delta)} \int_{0}^{\rho}(\rho-\sigma)^{\delta-1} h(\sigma) d \sigma .
$$

If $k \geqq 1, \beta>0, \delta>0, \eta<1 / k^{\prime}$, then

$$
\begin{aligned}
& \int_{0}^{1}(1-\rho)^{k, \beta-1} \rho^{k \eta-k \delta} H_{\delta}^{k}(\rho) d \rho \\
& \quad \leqq A(k, \beta, \delta, \eta) \int_{0}^{1}(1-\rho)^{k \beta+k \delta-1} \rho^{k \eta} h^{k}(\rho) d \rho .
\end{aligned}
$$

Choose $\mu, \omega$, depending on $k, \beta, \delta, \eta$, such that

$$
\delta / k^{\prime}<\mu<\beta+\delta / k^{\prime}, \quad \eta<\omega<1 / k^{\prime} .
$$

Writing $B$ for a constant depending on some or all of $k, \beta, \delta, \eta$, we obtain from Hölder's inequality and (15.3) that for $k>1$

(15.6) $\left\{\Gamma(\delta) H_{\delta}(\rho)\right\}^{k}$

$$
\begin{aligned}
& \leqq\left\{\int_{0}^{\rho}(1-\sigma)^{k \mu}(\rho-\sigma)^{\delta-1} \sigma^{k \omega} h^{k}(\sigma) d \sigma\right\}\left\{\int_{0}^{\rho}(1-\sigma)^{-k^{\prime} \mu}(\rho-\sigma)^{\delta-1} \sigma^{-k^{\prime} \omega} d \sigma\right\}^{k / k^{\prime}} \\
& \leqq B \rho^{k \delta / k^{\prime}-k \omega}(1-\rho)^{k \delta / k^{\prime}-k \mu} \int_{0}^{\rho}(1-\sigma)^{k \mu}(\rho-\sigma)^{\delta-1} \sigma^{k \omega} h^{k}(\sigma) d \sigma,
\end{aligned}
$$


since $\mu>\delta / k^{\prime}, \delta>0$ and $\omega<1 / k^{\prime}$. If $k=1$, the final inequality in (15.6) holds trivially (where $1 / k^{\prime}$ is interpreted as 0 ). Writing

$$
a=\delta+k \omega-k \eta, \quad c=k \beta+k \delta / k^{\prime}-k \mu,
$$

we therefore obtain from (15.6) and (15.4) that for $k \geqq 1$

$$
\begin{aligned}
\int_{0}^{1}(1- & \rho)^{k \beta-1} \rho^{k \eta-k \delta} H_{\delta}^{k}(\rho) d \rho \\
& \leqq B \int_{0}^{1}(1-\rho)^{c-1} \rho^{-a} d \rho \int_{0}^{\rho}(1-\sigma)^{k \mu}(\rho-\sigma)^{\delta-1} \sigma^{k \omega} h^{k}(\sigma) d \sigma \\
& \leqq B \int_{0}^{1}(1-\sigma)^{k k} \sigma^{k \omega} h^{k}(\sigma) d \sigma \int_{\sigma}^{1} \rho^{-a}(\rho-\sigma)^{\delta-1}(1-\rho)^{c-1} d \rho \\
& \leqq B \int_{0}^{1}(1-\sigma)^{k k^{\prime}+\delta+c-1} \sigma^{k \omega+\delta-a} h^{k}(\sigma) d \sigma,
\end{aligned}
$$

since $a>\delta>0, c>0$, and this is the required inequality.

The relation of Lemma 3 to the case $l=k$ of Theorem $\mathrm{B}$ can be seen by substituting $\rho=x /(1+x), \sigma=y /(1+y)$ in (15.5), and setting $f(x)=(1+x)^{-1-\delta} h(x /(1+x)), k \eta=-1-k \lambda, k \xi=k-k \beta-k \eta-1$. We thus obtain

$$
\begin{aligned}
\int_{0}^{+\infty}(1+x)^{k \xi} x^{-1-k \lambda-k \delta} F_{\delta}^{k}(x) d x & \\
& \leqq A(k, \delta, \lambda, \xi) \int_{0}^{+\infty}(1+x)^{k \xi} x^{-1-k \lambda} f^{k}(x) d x,
\end{aligned}
$$

where $k \geqq 1, \delta>0, \lambda>-1, \xi<1+\lambda$, and $F_{\delta}$ is defined as in Theorem B. For $\xi \leqq 0$ this is an immediate consequence of Theorem B with $f(x)$ replaced by $(1+x)^{\xi} f(x)$, but for $0<\xi<1+\lambda$ it requires an independent proof. There is presumably an extension of (15.7) with index $l \leqq k$ on the right, but we do not pursue this point.

Lemma 3 does not apply if $k=1, \eta=0$, and here we have an almost trivial result, namely

Lemma 4. Let $h, H_{\delta}$ be as in Lemma 3 , and let $\beta>0, \delta>0$. Then

$$
\int_{0}^{1}(1-\rho)^{\beta-1} H_{\delta}(\rho) d \rho \leqq A(\beta, \delta) \int_{0}^{1}(1-\rho)^{\beta+\delta-1} h(\rho) d \rho .
$$

Consider now the proof of Theorem 16. In view of the definition (14.4), it is enough to prove the inequality (15.1) when $0<\beta<\gamma<1$ and when $\beta \geqq 1$ and $[\beta] \leqq \beta<\gamma \leqq[\beta]+1$. It is therefore enough to prove it when $[\gamma]=[\beta]$ and when $\gamma=[\beta]+1$.

If $[\gamma]=[\beta]$, then, by $(14.5)$,

$$
\left|D^{\beta} \varphi\left(\rho e^{i \theta}\right)\right| \leqq \frac{1}{\Gamma(\gamma-\beta)} \int_{0}^{\rho}(\rho-\sigma)^{\gamma-\beta-1}\left|D^{\gamma} \varphi\left(\sigma e^{i \theta}\right)\right| d \sigma .
$$


For $k=1$ we have only to apply Lemma 4 with $\delta=\gamma-\beta$. For $k>1$ we apply Lemma 3 with $\delta=\gamma-\beta, \eta=(\gamma-[\gamma])(1-1 / k)$; this gives

$$
\begin{aligned}
& \int_{0}^{1}(1-\rho)^{k \beta-1} \rho^{(k-1)(\beta-[\beta])-\delta}\left|D^{\beta} \varphi\left(\rho e^{i \theta}\right)\right|^{k} d \rho \\
& \quad \leqq A(k, \beta, \gamma) \int_{0}^{1}(1-\rho)^{k \gamma-1} \rho^{(k-1)(\gamma-[\gamma])}\left|D^{\gamma} \varphi\left(\rho e^{i \theta}\right)\right|^{k} d \rho,
\end{aligned}
$$

and this obviously implies (15.1) for this case.

If $\gamma=[\beta]+1$, then (14.5) gives

$$
\left|D^{\beta} \varphi\left(\rho e^{i \theta}\right)\right| \leqq A(\beta)\left|c_{[\beta]}\right| \rho^{-(\beta-[\beta])}+\frac{1}{\Gamma(\gamma-\beta)} \int_{0}^{o}(\rho-\sigma)^{\gamma-\beta-1}\left|D^{\gamma} \varphi\left(\sigma e^{i \theta}\right)\right| d \sigma .
$$

Here we have only to apply Lemma 3 with $\eta=0$, and again we obtain the required result.

16. Lemma 3 enables us also to prove a theorem similar to Theorem 16 for the function $g_{k, \beta}$ defined by (5.5).

THEOREM 17. If $k>1, \gamma>\beta>0$, then for each $\theta$

$$
g_{k, \beta}(\theta) \leqq A(k, \beta, \gamma) g_{k, \gamma}(\theta) \text {. }
$$

Let $\delta=\gamma-\beta$. It is clearly enough to prove (16.1) when $\delta \leqq 1$. Since $\vartheta^{\beta} \varphi=\vartheta_{\hat{\delta}}\left(\vartheta^{r} \varphi\right)$, we then have (exactly as in the proof of Theorem 1)

$$
\rho^{\delta-1}\left|\vartheta^{\beta} \varphi\left(\rho e^{i \theta}\right)\right| \leqq \frac{1}{\Gamma(\delta)} \int_{0}^{o}(\rho-\sigma)^{\delta-1} \sigma^{-1}\left|\vartheta \gamma \varphi\left(\sigma e^{i \theta}\right)\right| d \sigma .
$$

Applying now Lemma 3 with $\eta=0$, we obtain (16.1).

17. In view of Theorem 16 , the argument of $\$ 7$ can be applied to $G_{k, \beta}$, and gives a result corresponding to Theorem 3. However, we can cover a number of such cases by using Theorem 3 directly, and we conclude with a proof of this. There are similar analogues of Theorems 4, 9 and 10.

TheOREM 18. Let $p>0, \beta>0, \mu=\max \{0,1 / p-1\}$, and let $\left(d_{n}\right)$ be a sequence of numbers such that, as $n \rightarrow \infty$

$$
d_{n}=n^{\beta} \sum_{\nu=0}^{m} \alpha_{\nu} n^{-\nu}+O\left(n^{\beta-m-1}\right),
$$

where $m$ is a fixed integer such that $m>\mu$, and $\alpha_{0}, \cdots, \alpha_{m}$ are fixed numbers. Let also $\varphi$ be defined as usual, let $\chi(z)=\sum_{n=1}^{\infty} c_{n} d_{n} z^{n}$, and let 


$$
\Gamma_{k, \beta}(\theta)=\left\{\int_{0}^{1}\left(\log \frac{1}{\rho}\right)^{k \beta-1} c(\rho)\left|\chi\left(\rho e^{i \theta}\right)\right|^{k} \frac{d \rho}{\rho}\right\}^{1 / k},
$$

where $c$ is bounded on the interval $[\delta, 1]$ for $0<\delta<1$, and the integral in (17.2) is convergent at 0 . Then for $k \geqq 2$

$$
\mathscr{C l}_{p}\left(\Gamma_{k, \beta}\right) \leqq A(k, p, \beta) \mathscr{C l}_{p}(\varphi) \text {. }
$$

We may obviously suppose that $\varphi(0)=c_{0}=0$. Let $C=\mathscr{C l}_{p}(\varphi)$, let $B$ denote a constant depending on some or all of $k, p, \beta$, and write

$$
\Gamma_{k, \beta}^{k}(\theta)=\int_{0}^{1 / 2}+\int_{1 / 2}^{1}=J_{1}+J_{2} .
$$

By Theorem D, $\left|c_{n}\right| \leqq B C_{n^{\mu}}$, and since $\left|d_{n}\right| \leqq A n^{\beta}$, it follows that $\left|\chi\left(\rho e^{i \theta}\right)\right| \leqq B C \rho$ for $0 \leqq \rho \leqq 1 / 2$, whence also $J_{1} \leqq B C^{k}$.

Next, by (17.1), we can write

$$
\chi=\sum_{\nu=0}^{m} \alpha_{\nu} \vartheta^{\beta-\nu} \varphi+\xi=\sum_{\nu=0}^{m} \alpha_{\nu} \vartheta^{\beta}\left(\vartheta_{\nu} \varphi\right)+\xi .
$$

Here

$$
\left|\xi\left(\rho e^{i \theta}\right)\right| \leqq A \sum_{n=1}^{\infty} n^{\beta-m-1}\left|c_{n}\right| \rho^{n} \leqq B C \sum_{n=1}^{\infty} n^{\beta+\mu-m-1} \rho^{n} \leqq B C(1-\rho)^{-1}
$$

if $\beta+\mu-m \neq 0$, where $\gamma=\max \{0, \beta+\mu-m\}$, and

$$
\left|\xi\left(\rho e^{i \theta}\right)\right| \leqq B C \log (1 /(1-\rho))
$$

if $\beta+\mu-m=0$. Hence in either case

$$
\int_{1 / 2}^{1}\left(\log \frac{1}{\rho}\right)^{k \beta-1}\left|\xi\left(\rho e^{i \theta}\right)\right|^{k} \frac{d \rho}{\rho} \leqq B C^{k}
$$

Using Theorem 3 , it follows now that

$$
\mathscr{C}_{p}\left(\Gamma_{k, \beta}\right) \leqq B C+B \sum_{\nu=0}^{m}\left|\alpha_{\nu}\right| \mathscr{C l}_{p}\left(\vartheta_{\nu} \varphi\right) \text {. }
$$

A simple argument shows also that

$$
\mathscr{C}_{p}\left(\vartheta_{\nu} \varphi\right) \leqq \mathscr{C}_{p}(\varphi) \quad(\nu=1,2, \cdots),
$$

and this completes the proof.

\section{REFERENCES}

1. A. Erdelyi (ed), Higher transcendental Functions, Vol. 1, New York, 1953.

2. T. M. Flett, On some theorems of Littlewood and Paley, J. London Math. Soc. 31 (1956), 336-44. 
3. - On an extension of absolute summability and some theorems of Littlewood and Paley, Proc. London Math. Soc. (3) 7 (1957), 113-41.

4. - Some more theorems concerning the absolute summability of Fourier series and power series, Proc. London Math. Soc. (3) 8 (1958), 357-87.

5. — A note on some inequalities, Proc. Glasgow Math. Assoc. 4 (1958), 7-15.

6. - Some theorems on fractional integrals, Proc. Camb. Phil. Soc. 55 (1959), $31-50$.

7. J. Hadamard, Essai sur l'étude des fonctions données par leur developpement de Taylor, Journal de Math. (4) 8 (1892), 101-186.

8. G. H. Hardy and J. E. Littlewood, Some new properties of Fourier constants, Math. Ann. 97 (1926), 159-209.

9. Notes on the theory of series (V): On Parseval's theorem, Proc. London Math. Soc. (2) 26 (1927), 287-94.

10. Elementary theorems concerning power series with positive coefficients and moment constants of positive functions, Journal für Math. 157 (1927), 141-58.

11. - A convergence criterion for Fourier series, Math. Zeitschrift 28 (1928), 612-34.

12. - Some properties of fractional integrals II, Math. Zeitschrift 34 (1932), 403-39.

13. - Some new cases of Parseval's theorem, Math. Zeitschrift 34 (1932), 620-33.

14. - An additional note on Parseval's theorem, Math. Zeitschrift 34 (1932), 634-36.

15. - Notes on the theory of series (XIX). A problem concerning majorants of Fourier series, Quart. J. Math. 6 (1935), 304-15.

16. Notes on the theory of series (XXI). Generalizations of a theorem of Paley, Quart. J. Math. 8 (1937), 161-71.

17. Theorems concerning mean values of analytic or harmonic functions, Quart. J. Math. 12 (1941), 221-256.

18. I. I. Hirschman, Fractional integration, Amer. J. Math. 75 (1953), 531-46.

19. J. E. Littlewood and R.E.A.C. Paley, Theorems on Fourier series and power series (II), Proc. London Math. Soc. (2) 42 (1936), 52-89.

20. J. Marcinkiewicz and A. Zygmund, A theorem of Lusin, Duke Math. J. 4 (1938), 473-85.

21. M. Weiss, On Hardy-Littlewood series, Trans. Amer. Math. Soc. 91 (1959), $470-479$.

22. A. Zygmund, Trigonometric Series, 2 vols., Cambridge, 1959.

Received February 23, 1967.

THE UNIVERSITY OF SHEFFIELD AND

THE UNIVERSITY OF WASHINGTON 


\title{
PACIFIC JOURNAL OF MATHEMATICS
}

\author{
EDITORS
}

\section{H. ROYDEN}

Stanford University

Stanford, California

\author{
J. P. JANS \\ University of Washington \\ Seattle, Washington 98105
}

\section{J. DugundJI}

Department of Mathematics

University of Southern California

Los Angeles, California 90007

\section{RICHARD ARENS}

University of California

Los Angeles, California 90024

\section{ASSOCIATE EDITORS}
E. F. BECKENBACH
B. H. NEUMANN
F. WOLF
K. YOSIDA

\section{SUPPORTING INSTITUTIONS}

\author{
UNIVERSITY OF BRITISH COLUMBIA \\ CALIFORNIA INSTITUTE OF TECHNOLOGY \\ UNIVERSITY OF CALIFORNIA \\ MONTANA STATE UNIVERSITY \\ UNIVERSITY OF NEVADA \\ NEW MEXICO STATE UNIVERSITY \\ OREGON STATE UNIVERSITY \\ UNIVERSITY OF OREGON \\ OSAKA UNIVERSITY \\ UNIVERSITY OF SOUTHERN CALIFORNIA
}

\author{
STANFORD UNIVERSITY \\ UNIVERSITY OF TOKYO \\ UNIVERSITY OF UTAH \\ WASHINGTON STATE UNIVERSITY \\ UNIVERSITY OF WASHINGTON \\ AMERICAN MATHEMATICAL SOCIETY \\ CHEVRON RESEARCH CORPORATION \\ TRW SYSTEMS \\ NAVAL WEAPONS CENTER
}

Mathematical papers intended for publication in the Pacific Journal of Mathematics should be in typed form or offset-reproduced, double spaced with large margins. Underline Greek letters in red, German in green, and script in blue. The first paragraph or two must be capable of being used separately as a synopsis of the entire paper. It should not contain references to the bibliography. Manuscripts, in duplicate if possible, may be sent to any one of the four editors. All other communications to the editors should be addressed to the managing editor, Richard Arens, University of California, Los Angeles, California 90024.

Each author of each article receives 50 reprints free of charge; additional copies may be obtained at cost in multiples of 50 .

The Pacific Journal of Mathematics is published monthly. Effective with Volume 16 the price per volume (3 numbers) is $\$ 8.00$; single issues, $\$ 3.00$. Special price for current issues to individual faculty members of supporting institutions and to individual members of the American Mathematical Society: $\$ 4.00$ per volume; single issues $\$ 1.50$. Back numbers are available.

Subscriptions, orders for back numbers, and changes of address should be sent to Pacific Journal of Mathematics, 103 Highland Boulevard, Berkeley 8, California.

Printed at Kokusai Bunken Insatsusha (International Academic Printing Co., Ltd.), 7-17, Fujimi 2-chome, Chiyoda-ku, Tokyo, Japan.

PUBLISHED BY PACIFIC JOURNAL OF MATHEMATICS, A NON-PROFIT CORPORATION

The Supporting Institutions listed above contribute to the cost of publication of this Journal, but they are not owners of publishers and have no responsibility for its content or policies. 


\section{Pacific Journal of Mathematics}

\section{Vol. 25, No. $3 \quad$ November, 1968}

Philip Marshall Anselone and Theodore Windle Palmer, Collectively

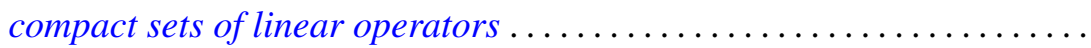

Philip Marshall Anselone and Theodore Windle Palmer, Spectral analysis of collectively compact, strongly convergent operator sequences.........

Edward A. Bender, Characteristic polynomials of symmetric matrices...... 433

Robert Morgan Brooks, The structure space of a commutative locally convex

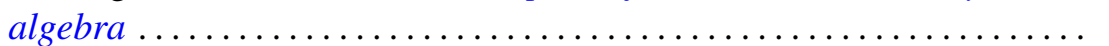

Jacob Feldman and Frederick Paul Greenleaf, Existence of Borel

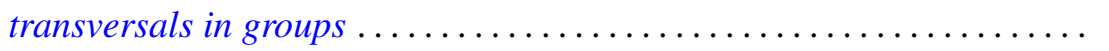

Thomas Muirhead Flett, Mean values of power series 463

Richard Vernon Fuller, Relations among continuous and various

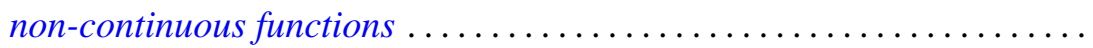

Philip Hartman, Convex sets and the bounded slope condition ............

Marcel Herzog, On finite groups containing a CCT-subgroup with a cyclic Sylow subgroup .

James Secord Howland, On the essential spectrum of Schroedinger

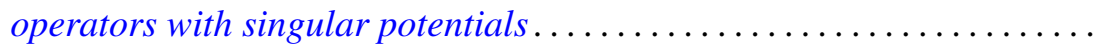

Thomas William Hungerford, On the structure of principal ideal rings .....

Paul Joseph Kelly and Ernst Gabor Straus, Curvature in Hilbert geometries. II mpati Madhusudana Rao, Linear functionals on Orlicz spaces:

Malempati Madhusudana Rao, Linear functionals on Orli
$\quad$ General theory............................
Stanley F. Robinson, Theorems on Brewer sums.......
Ralph Tyrrell Rockafellar, A general correspondence betw

Malempati Madhusudana Rao, Linear functiona
$\quad$ General theory.........................
Stanley F. Robinson, Theorems on Brewer sums
Ralph Tyrrell Rockafellar, A general corresponden

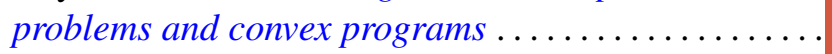

Richard Benjamin Sher, Defining subsets of $E^{3}$ by cubes.

Howard Jacob Weiner, Invariant measures and Cesàro summability... 